\title{
A network pharmacology study on the Cervix Prescription for treatment of Cervical Cancer
}

\section{Pian Ying}

The First Affiliated Hospital of Zhejiang University of Traditional Chinese Medicine

Yingping Zhu ( $\nabla$ zhuyingping_26@126.com )

The First Affiliated Hospital of Zhejiang University of Traditional Chinese Medicine

\section{Research Article}

Keywords: network pharmacology, Qiu's Cervical Formula, cervical cancer, molecular docking

Posted Date: August 24th, 2021

DOl: https://doi.org/10.21203/rs.3.rs-835232/v1

License: (c) (i) This work is licensed under a Creative Commons Attribution 4.0 International License. Read Full License 


\title{
A network pharmacology study on the Cervix Prescription for treatment of Cervical Cancer
}

\author{
Yingpian,Zhu Yingping1* \\ (The First Affiliated Hospital of Zhejiang University of Traditional Chinese \\ Medicine, Hangzhou 310006, China)
}

\begin{abstract}
Purpose: Based on the method of network pharmacology to explore the mechanism of the Cervical Prescription(CP) in the treatment of cervical cancer(CC).

Methods: To obtain the active ingredients and potential targets of $\mathrm{CP}$ from the Traditional Chinese Medicine Systems Pharmacology database and analysis platform (TCMSP) ; To search for targets related to cervical cancer through the database, and map the CP and the targets; Screen the core Targets, a Chinese medicine compoundtarget network and STRING database was used to construct a protein-protein interaction network (PPI). The DAVID 6.8 online tool was used to perform gene ontology (GO) and kyoto encyclopedia of genes and genome (KEGG) pathway enrichment analyses of overlapping targets.
\end{abstract}

Results: The $\mathrm{CP}$ contains 2 active ingredients, corresponding to 301 non-reactive targets; $10 \mathrm{GO}$ biological process related items and 73 signal pathways were obtained.

Conclusion: By constructing a network diagram of "components-targets-pathways",

\footnotetext{
${ }^{1}$ Project fund: Zhejiang Provincial Natural Science Foundation Project(CN) (LQ20H270016); Zhejiang Traditional Chinese Medicine Administration (CN) (2020ZB087).

*Profile of the author: Zhu Yingping, (1987-), female, attending physician, from Ganzhou, Jiangxi Province, master's degree student, research direction: Chinese medicine prevention and treatment of gynecological malignancies; E-mail: zhuyingping_26@126.com
} 
this study revealed that $\mathrm{CP}$ can exert its efficacy through multiple components, multiple targets, and multiple pathways to achieve the purpose of preventing and treating CC.The network pharmacology-based approach in the present study showed promising potential for identifying the main therapeutic targets from TCM formulas. This study provides valuable information for TCM researchers and clinicians to better understand the main therapeutic targets and therapeutic roles of herbal decoctions in clinical settings.

Keywords: network pharmacology,Qiu's Cervical Formula,cervical cancer,molecular docking

\section{Background}

Cervical cancer is the second most common malignancy that affects women, ranking after breast cancer (1). There are about 75,000 new cases and 33,000 deaths in China each year, accounting for about 1/10 of the world's total cases(2). Modern studies have confirmed that human papillomavirus (HPV) infection is closely related to the occurrence, development, treatment and prevention of cervical cancer. At present, western medicine is mainly used for regular monitoring of HPV-susceptible and infected individuals to prevent and reduce the occurrence of cervical cancer. The treatment strategies of cervical cancer mainly includes surgery, radiotherapy and chemotherapy. However, there is still a lack of treatment strategies for HPV infection in the cervix and vagina, and so effective treatment approaches should be determined(3). By adjusting the immune function of the body, Chinese medicine has shown several irreplaceable advantages in improving the clinical symptoms of cancer 
patients as well as the quality of life of patients (4). With the advancements in medical technology, the research on traditional Chinese medicine (TCM) in the field of antivirals is increasing. Recently, TCM has expanded its thinking on preventing and treating cervical cancer as well as cervical HPV infection.

TCM has the advantages of individualized treatment and multiple effective ingredients and multiple targets, with good developmental prospects. However, Chinese medicine therapy is like a black box, in which the specific mechanism and target of the drug's action remain unclear, hindering the improvement of its clinical efficacy.

Network pharmacology is suitable for characterizing multi-component, multitarget, and multi-pathways of Chinese medicine. To further clarify the effectiveness of Chinese medicine compatibility in many aspects(5), this article adopted the method of network pharmacology and started with the idea of "molecular-target-pathway". A network model with regard to components, targets, protein interactions, biological functions and signaling pathways of "Cervical Recipe" was drawn, the potential relationship in the network system was analyzed, and the pharmacology methodology method was further clarified.

\section{Methods}

\section{Analysis of effective ingredients contained in Cervical Prescription}

Cervical Prescription contains 30g of alum, catechin, and $1 \mathrm{~g}$ borneol. The three medicines are mashed and mixed with sesame oil for use. The BATMAN-TCM database(6) was used to search these three Chinese medicines, found the compounds 
contained in them as effective ingredients, and deduplicated the effective ingredients in these Chinese medicines.

\section{Analysis of potential targets of Cervical Prescription}

Based on the interaction relationship between the compound and the target protein in the BATMAN-TCM database and the interaction score, the target protein of the active ingredients in all the traditional Chinese medicines (TCMs) is considered as a potential target of Gong Jing Fang. The BATMAN-TCM database involves all known compound-target protein interactions, and combines the structural similarity of the compound and the structural similarity of the protein (including the sequence similarity of the protein) to predict the target interaction of the compound and the protein that causes interaction. The database comprises of most of the interaction relationships in the field of TCM target prediction. After obtaining the effective components of TCM in this analysis, BATMAN-TCM was used to analyze and screen the targets of the compounds, with target interaction scores of $\geq 20$ as potential targets of Cervical Prescription.

\section{Analysis of cervical cancer related targets}

Using "cervical cancer" as a keyword, the following 5 databases were searched to obtain the known drug targets and the most relevant or most likely drug targets for cervical cancer. The Kyoto Encyclopedia of Genes and Genome (KEGG) database(7): the KEGG pathway is the most famous database of all. The KEGG database was searched for cervical cancer and the sub-database KEGG disease was selected to obtain a list of molecules whose molecular interference leads to the occurrence and 
development of the disease. The molecules in the list can be used as targets for cervical cancer prevention and treatment.

PHARMGKB database(8): This is one of the most important databases in the field of pharmacogenomics. It contains most of the complete pharmacogenomic and phenotypic (including various diseases) information, as well as data on pharmacodynamics and pharmacokinetics. The term "cervical cancer" was used to search in this database to obtain drug target molecules of this. Comparative Toxicogenomics Database (CTD)(9): This database is based on literature and manually corrected. It records the detailed links between compounds, gene products, phenotypes, diseases, and environmental exposures. One of its important functions involves correlating between the disease and the gene/protein and its correlation score. The term "cervical cancer" was searched in the database to obtain potential target molecules of this, and further the genes with correlation $\geq 50$ were screened as potential target molecules for cervical cancer.

GeneCards database(10): This is one of the comprehensive databases on genes, which include the association between genes and diseases. The term "cervical cancer" was searched in this database to obtain cervical cancer-related genes (provide correlation scores between genes and diseases from multiple aspects), and further the genes with correlation scores $\geq 10$ points were screened. These genes can be used as potential target molecules for cervical cancer. DisGeNET database(11): This database is one of the largest databases used for identifying the association of diseases and gene/gene isomers. It integrates expert manual correction, genome-wide association 
studies (GWAS) high-throughput omics data, animal models and literature reports data. The database is not only publicly available, but also provides a cytoscape plugin for querying. The term "cervical cancer" was searched in this database to obtain potential targets of cervical cancer. By combining with the cervical cancer targets obtained from the above 5 databases and deduplication was performed to obtain the final cervical cancer target molecules.

\section{Traditional Chinese Medicine-Ingredients-Target Analysis}

After obtaining the information regarding the active ingredients of all TCMs, the active ingredient-target interaction information was combined with the "Chinese medicine compound-component-target" network to construct Cervical Prescription. Further the network of "Chinese medicine compound-components-targets" was screened, and only the targets in the network that are also targets of cervical cancer target molecules were retained (at the same time remove the effective components that have no interaction targets caused by deletion of the targets), and finally obtain an effective "Chinese medicine compound-component-target" network. Next, a cytoscape(12)(version 3.7.1) software was used to visualize the network. At the same time, the genes in the traditional Chinese medicine-component-target network are extracted for downstream analysis.

\section{Protein Interaction (PPI) Network Analysis}

The STRING (https://string-db.org/) online database(13) was used for obtaining the target gene list to analyze the protein-protein interaction (PPI). In this analysis, the confidence of protein interactions was set to medium confidence, i.e, only protein 
interactions with combined scores of $>0.4$ are retained. After obtaining the PPI network, Cytoscape software was used to visualize and analyze the PPI network. The vast majority of biological networks are scale-free (scale-free) networks. The typical feature is that most of the nodes in the network are connected to only a few nodes, and there are very few nodes that are connected to a very large number of nodes. The nodes that are connected by many nodes are important in the network, especially for biological networks, in which these nodes play as key genes (hub genes), and are likely to participate in the regulation of many biological processes. For larger interactive networks, based on network statistical analysis, the cytohubba plug-in(14) was used to analyze the degree and betweenness of nodes, and the nodes whose connectivity and betweenness are greater than the average value are selected as the Key target compounds. These key targets are more likely to have the effects of preventing and treating cervical cancer, and deserve more research attention when compared with other target genes.

\section{Gene function enrichment analysis}

This analysis used the Gene Ontology database(15) and KEGG pathway database(7) to perform functional enrichment analysis on the target gene list. A statistical algorithm (Fisher's exact test) was used to find out as to which GO TERM/pathway has the greatest correlation between a set of genes and the analysis results. Each item in the analysis results has a statistical value of P-value to indicate its significance. The smaller the p-value is, the higher the degree of enrichment of gene list in this GO TERM/pathway, and more likely the gene list affects the life 
activities of cells through this GO TERM/pathway, which is worthy of further study.

\section{Survival analysis}

The target gene list was selected and the clinical data and gene expression data were combined in the TCGA-CESC to analyze the genes whose expression and prognosis showed significant relation to the gene set. By using the median gene expression as the grouping limit, the samples were divided into two groups of high expression and low expression. The COX method was used to analyze the survival differences between the two groups of samples (overall survival difference and disease-free survival difference), and a survival curve was drawn. At the same time, the correlation between gene expression in the list and other clinical parameters (such as case staging) was analyzed to draw a forest plot.

\section{Results}

\section{Cervix Prescription active ingredients and their targets}

Cervical Prescription contains 3 TCMs, 2 were obtained from the BATMANTCM database, in which alum is not recorded in the BATMAN-TCM database, and sesame oil as a preparation is not recorded in the BATMAN-TCM database.

Catechu contains 17 active ingredients, and 8 active ingredients had structural information. After screening by target interaction $\geq 20$ points, there are 4 active ingredients in this, and these have a total of 36 non-repetitive interaction targets.

Borneol contains 32 active ingredients, and 17 active ingredients had structural information. After screening for target interaction $\geq 20$ points, there are 15 active 
ingredients in borneol, and these have a total of 272 non-repetitive interaction targets. Combining the active ingredients of all TCMs in Cervical Prescription (with $\geq 20$ points of active ingredients that interact as targets) and interactive targets, 17 kinds of non-repetitive active ingredients and 301 non-repetitive interactive targets were obtained.For specific statistics, see Table 1 below:

Table 1 Compatibility of Traditional Chinese Medicine Compounds-Active Ingredients-Target Statistics

\begin{tabular}{lccc}
\hline & $\begin{array}{c}\text { Number of } \\
\text { active }\end{array}$ & Number of active & \\
& ingredients (after & Number of \\
Chinese medicine & ingredients & screening) & targets \\
\hline Catechu & $17 / 8$ & 4 & 36 \\
Borneol & $32 / 17$ & 15 & 272 \\
\hline
\end{tabular}

The potential target predictions of a total of 346 target genes related to new coronavirus pneumonia were collected from the GeneCards database. The Venny 2.1.0 tool was used to map and compare the potential target genes of drugs with disease genes and a Venn diagram was drawn (Figure 1), in which 49 common genes were obtained.

Cervical Prescription contains 19 non-repetitive active ingredients that have $\geq 20$ points of mutual targets, and there are a total of 301 non-repetitive drug targets. The intersection of all active ingredients in Cervical Prescription and their mutual targets was as follows: 

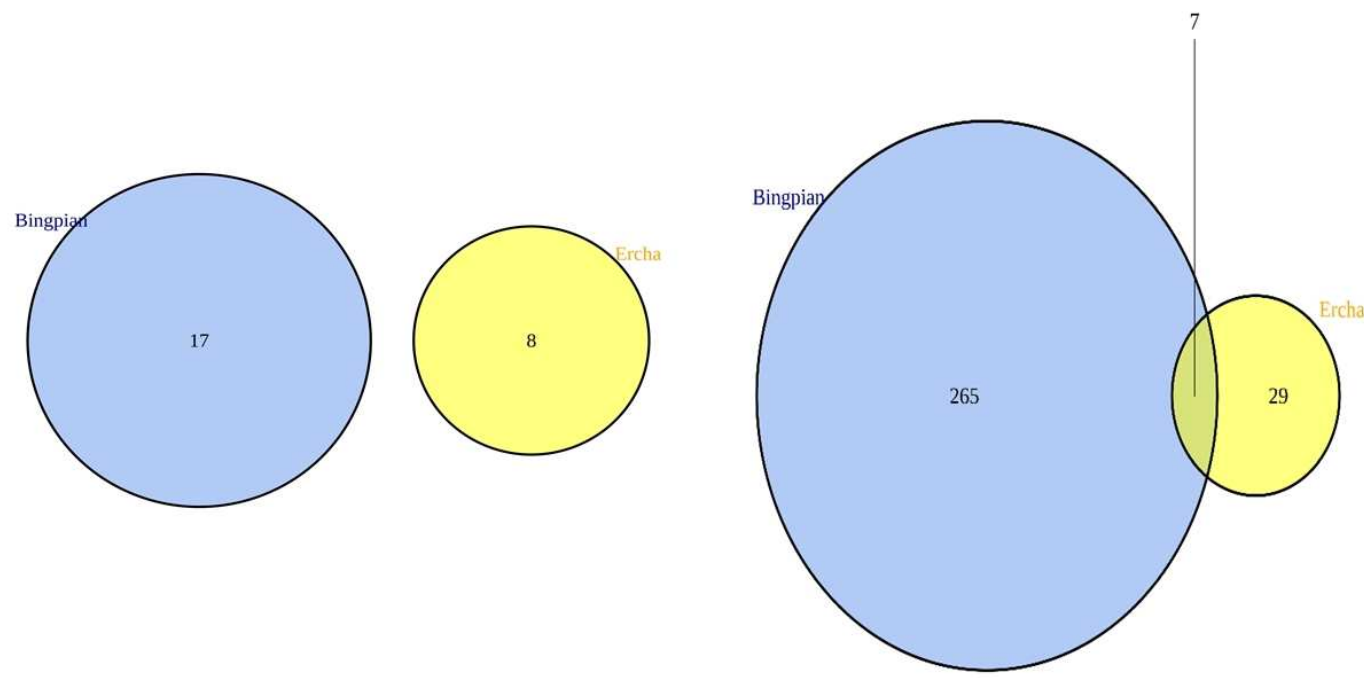

Figure 1 Interaction target intersection and union Venn diagram

All the active ingredients and target interaction genes were combined after screening for TCMs, and deduplication was performed to obtain non-repetitive effective ingredients and non-repetitive effective targets. The corresponding relationship between TCM and effective ingredients, and the corresponding relationship between effective ingredients and target was then observed.

\section{Analysis of cervical cancer related targets}

In the KEGG and PHARMGKB databases, there are 7 target molecules for cervical cancer.In the CTD database, there are 1,658 target molecules obtained for cervical cancer, and there are 1211 target molecules obtained for cervical cancer from the GeneCards database. In the DisGeNE database, there are 964 target molecules obtained for cervical cancer (Table 2).

Table 2 Five Target molecules of cervical cancer in the database 
The cervical cancer target molecules from the above 5 databases were combined to obtain 2,844 non-repetitive cervical cancer target molecules. The intersection set of cervical cancer targets from the 5 databases was as follows (Figure 2):

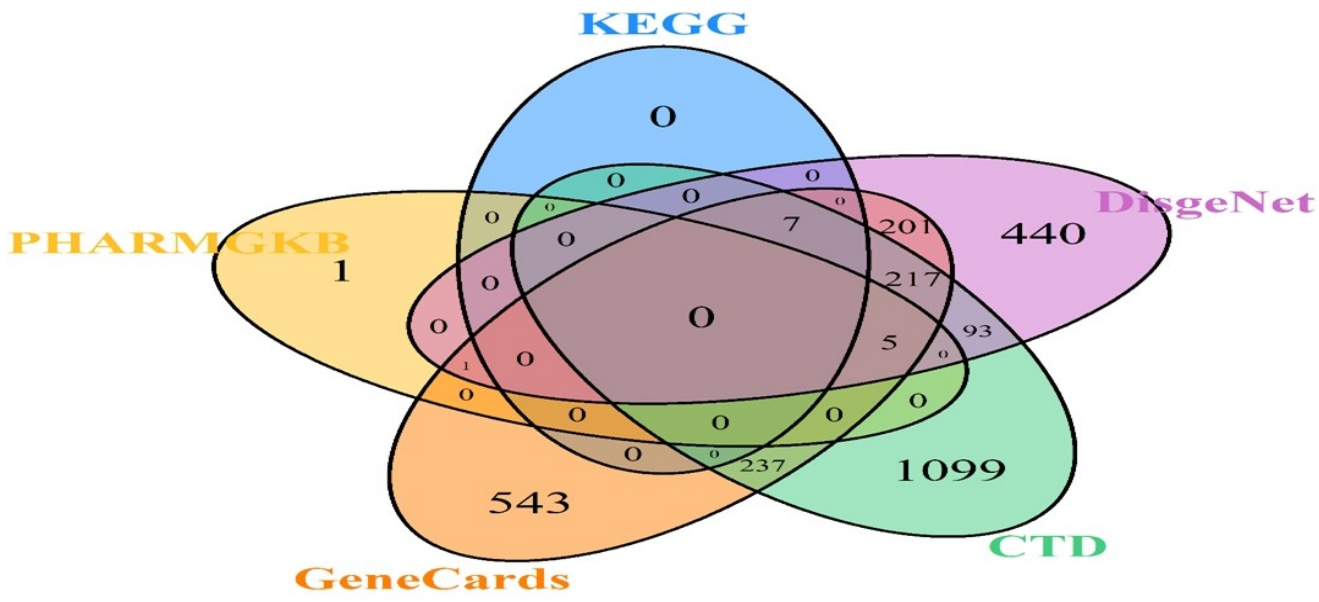

Figure 2 Five Potential cervical cancer targets in databases

\section{Traditional Chinese Medicine-Ingredients-Target Analysis}

The Chinese medicine-active ingredients and active ingredients-targets were combined to form a "Chinese medicine compound-components-targets" network, and combined with the cervical cancer target molecules for further screening, and cytoscape was used to visualize the following (Figure 3):Combine 2.1 traditional Chinese medicine-active ingredients and active ingredients-targets to form a "Chinese medicine compound-ingredients-targets" network, and combine with the cervical cancer target molecules in 2.2 for further screening, and use cytoscape to visualize it 
as follows (Figure 3):

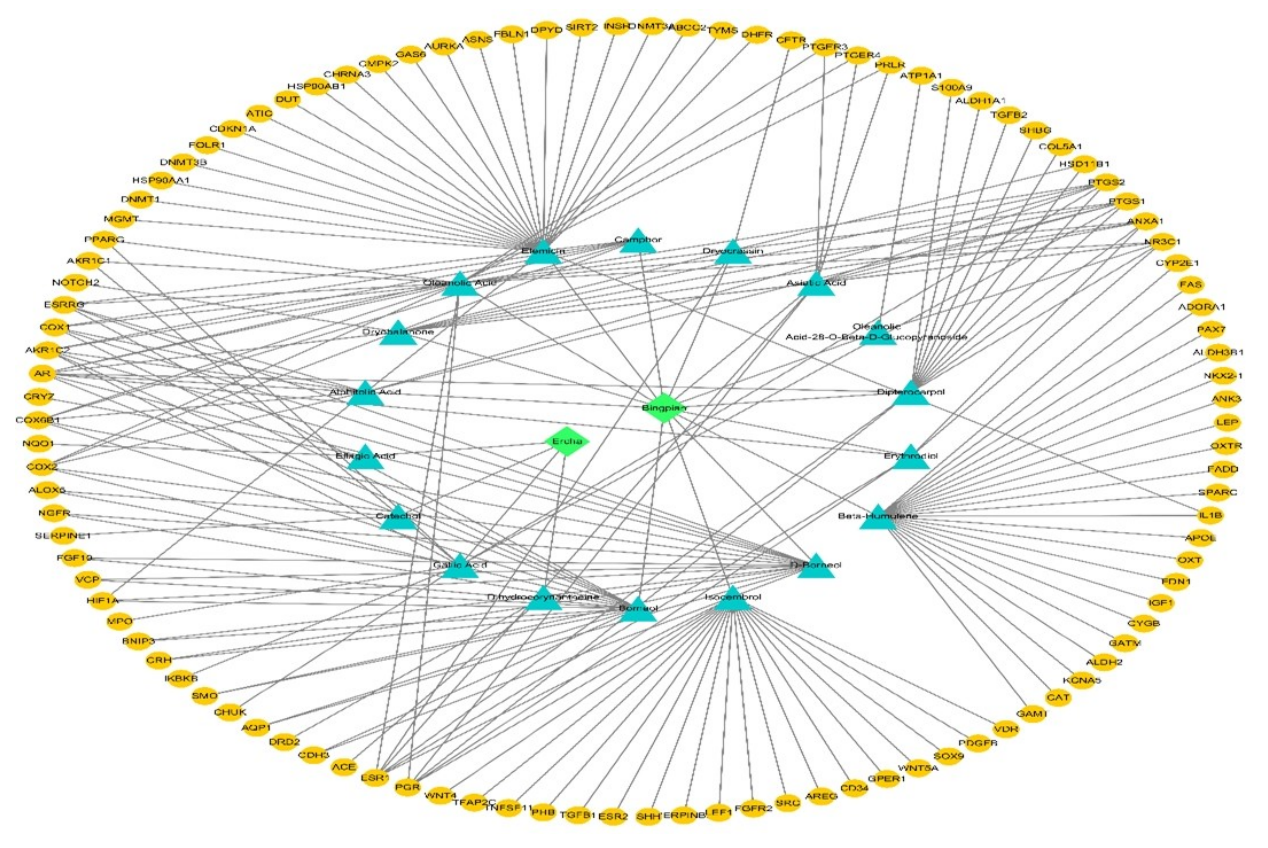

Figure3 Traditional Chinese Medicine Compound-Ingredients-Target Network

\section{PPI network analysis}

For the target genes in the traditional Chinese medicine compound-componenttarget network, the STRING database was used to analyze the PPI interactions to obtain the interaction pair. The cytoscape software was used to visualize it to obtain the following PPI network that has 106 nodes/proteins and 646 edges/interactions (Figure 4). 


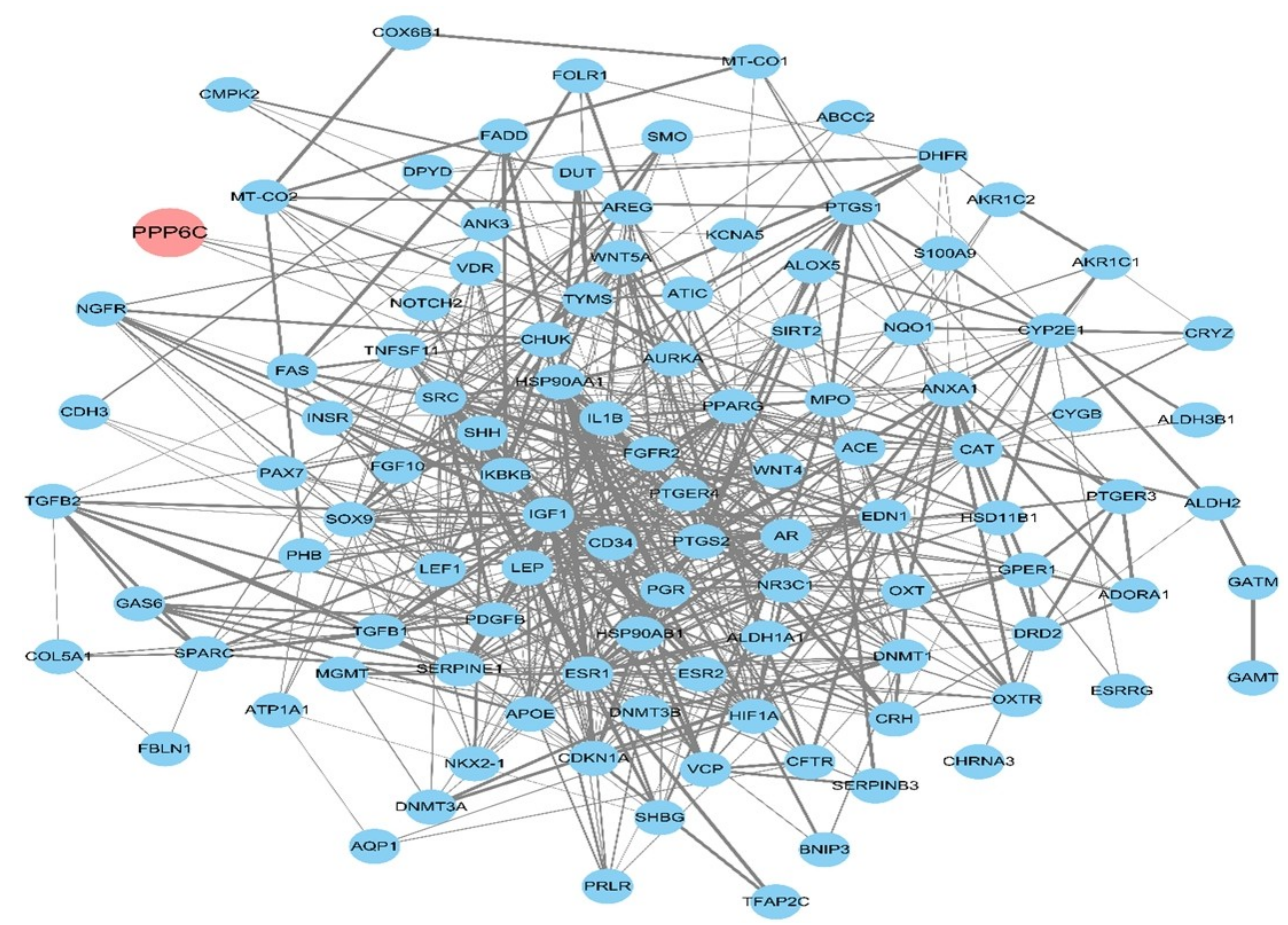

Figure4 PPI interaction network of cervical cancer target protein

The cytohubba plug-in was used to analyze the connectivity and mediation of nodes in the PPI network, and then the following top nodes can be obtained (Table 3):

\begin{tabular}{lcc}
\hline node_name & Degree & Betweenness \\
\hline ESR1 & 47 & 1227.13963 \\
IGF1 & 47 & 1001.55617 \\
SRC & 43 & 1193.39528 \\
IL1B & 39 & 859.49686 \\
PTGS2 & 39 & 593.08096 \\
\hline LEP & 30 & 379.44828 \\
PTGS2 & 19 & 25.42108 \\
\hline IGF1 & 18 & 13.16431 \\
\hline ESR1 & 18 & 18.68824 \\
\hline
\end{tabular}




\begin{tabular}{lcc}
\hline SRC & 17 & 27.1046 \\
HIF1A & 16 & 14.29995 \\
IL1B & 15 & 6.48085 \\
\hline
\end{tabular}

Table 3 The top nodes in the PPI network

The nodes whose connectivity and mediation degree are greater than the average value in the above-mentioned PPI interaction network are screened as key targets of Qiu's Cervical formula, and 23 key targets were obtained. The 23 targets constitute the following PPI interaction network, which has a total of 23 nodes/proteins and 147 edges/interactions (Figure 5).

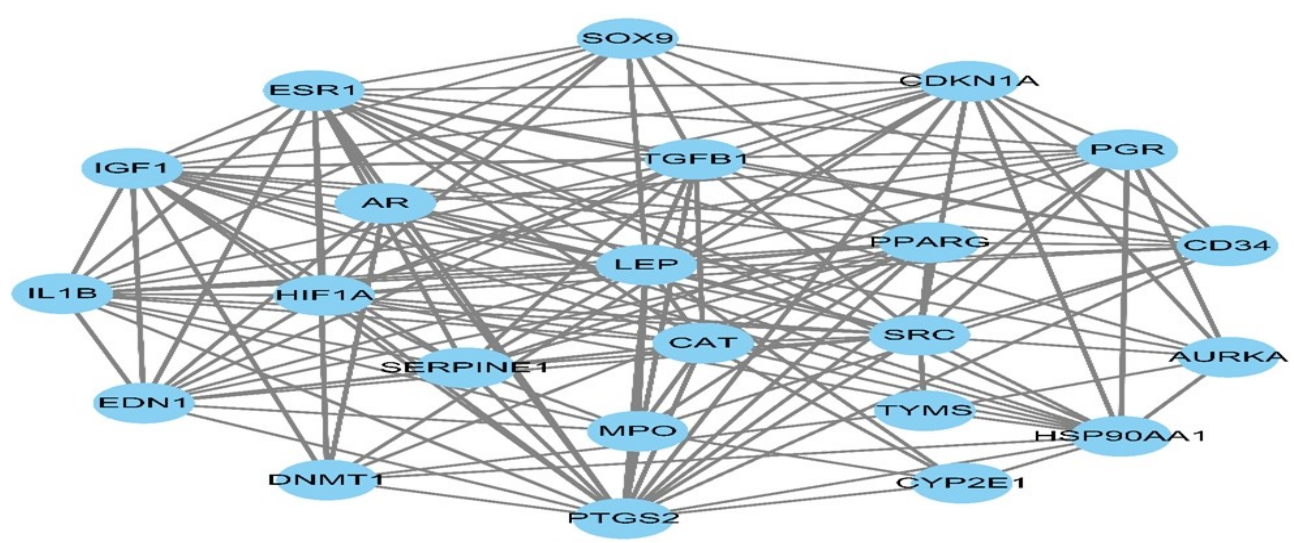

Figure 5 PPI interaction network of key target proteins of the compound

\section{Functional enrichment analysis}

Functional enrichment analysis of genes in the compound-component-target network of traditional Chinese medicine

The functional enrichment analysis on genes (plus PPP6C) was performed in the "Chinese medicine compound-component-target" network to analyze their enrichment in GO ontology and KEGG, and obtain the following enrichment results (Figure 6, Figure 7) 


\section{Significant GO-BP}

Response to organic substance

Response to lipid

Response to organic cyclic compound

Cellular response to organic substance

Response to hormone

Regulation of cell death

Negative regulation of cell death

Response to drug

Response to steroid hormone

Positive regulation of metabolic process

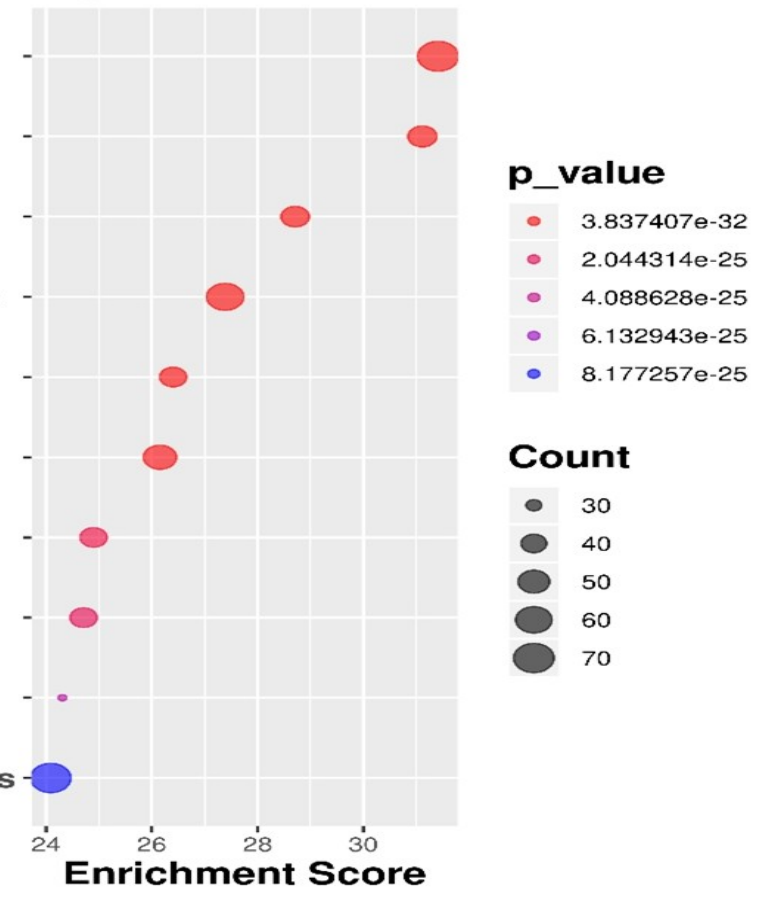

Figure $6 \mathrm{GO}$ enrichment analysis of genes in the network of "Chinese medicine compound-components-targets": bubble chart.

\section{Significant pathway}

Pathways in cancer

Antifolate resistance

Prostate cancer

Proteoglycans in cancer

Breast cancer

Chagas disease (American trypanosomiasis) -

Non-alcoholic fatty liver disease (NAFLD)

IL-17 signaling pathway

FoxO signaling pathway

Fluid shear stress and atherosclerosis

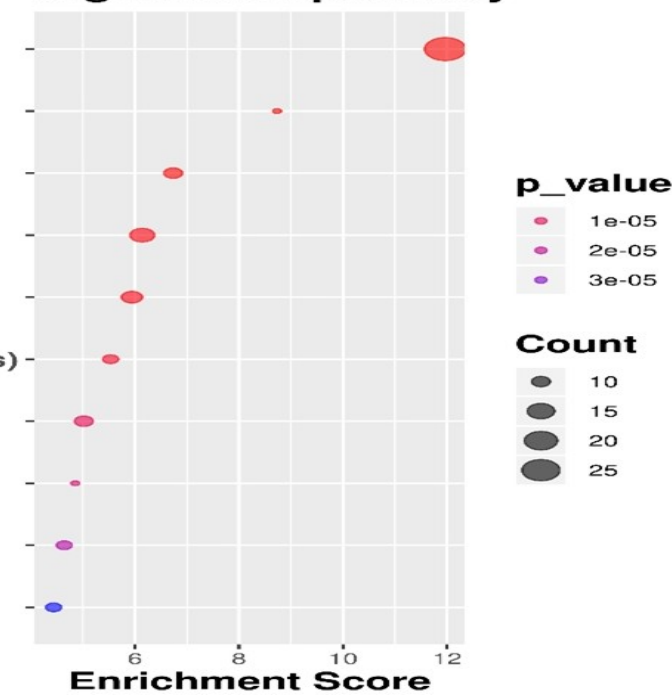

Figure 7 KEGG enrichment analysis of genes in the network of "Chinese

medicine compound-components-targets": bubble chart.

The pathview package was used to visualize some of the significantly enriched pathways, and the following pictures were obtained (Figure 8): 


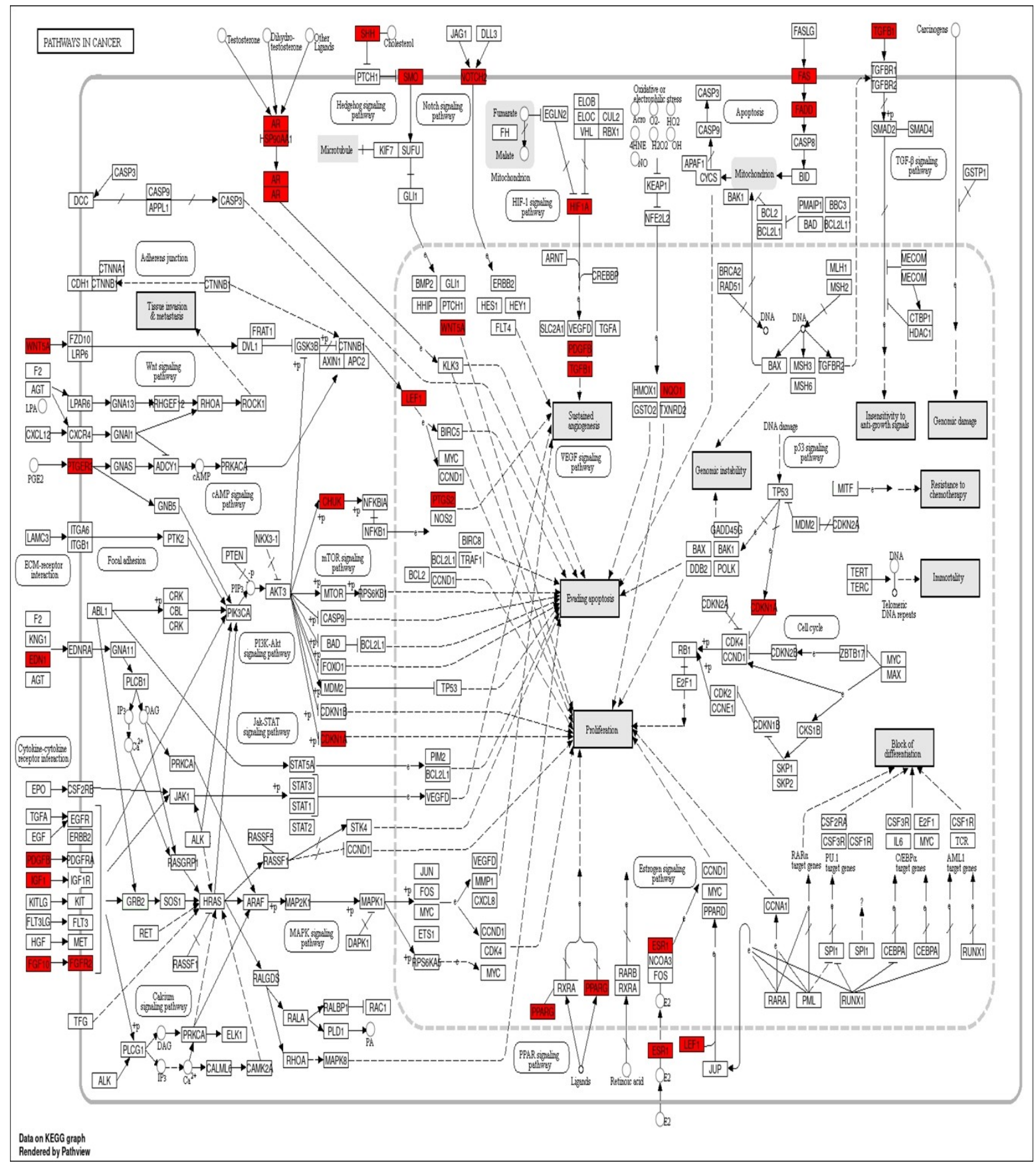

Figure 8 Pathway in cancer

\section{Functional enrichment analysis of compound key target genes}

Functional enrichment analysis on 23 compound key target genes was performed, and their enrichment was analyzed in GO ontology and KEGG. The following enrichment results were obtained (Figure 9, Figure 10): 


\section{Significant GO-BP}

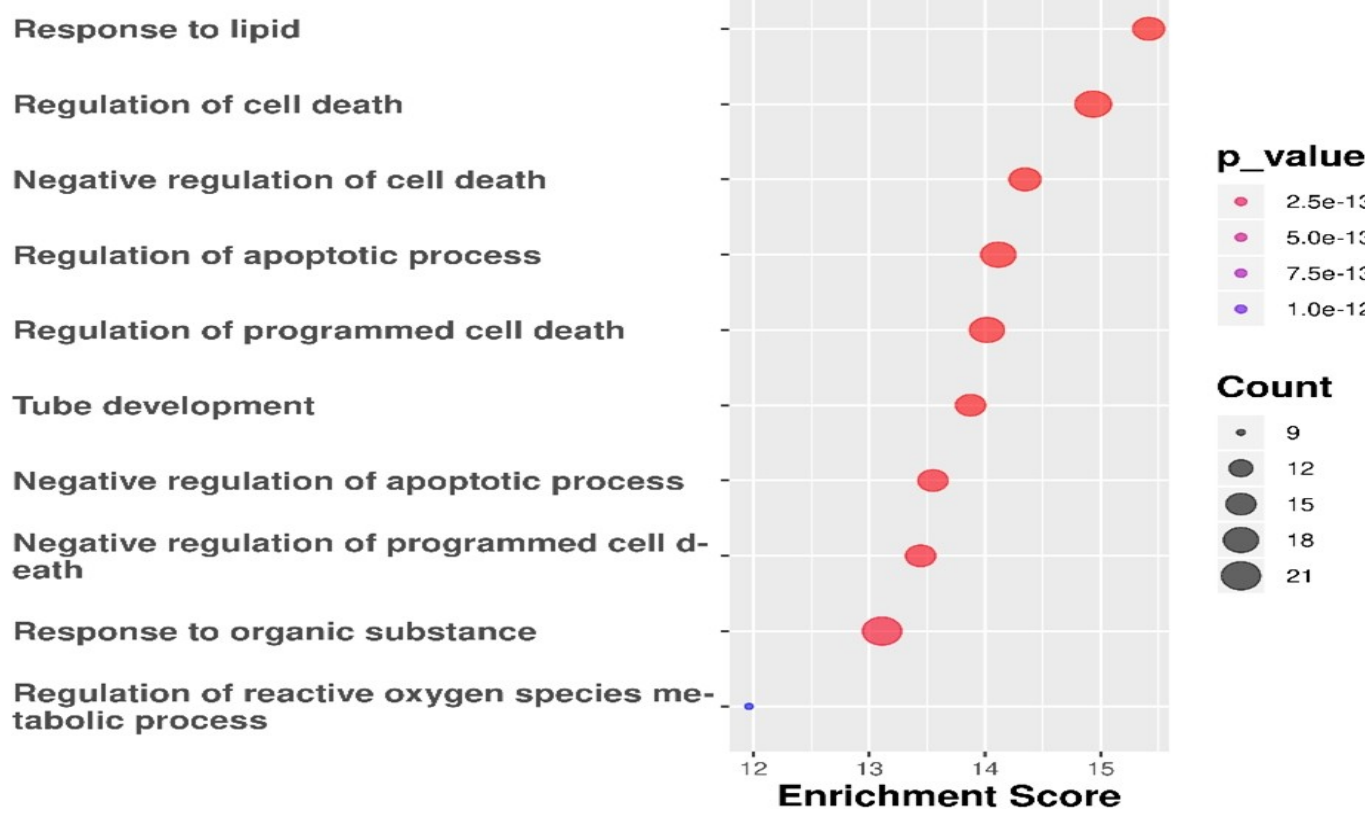

Figure 9 Bubble chart of GO enrichment analysis.

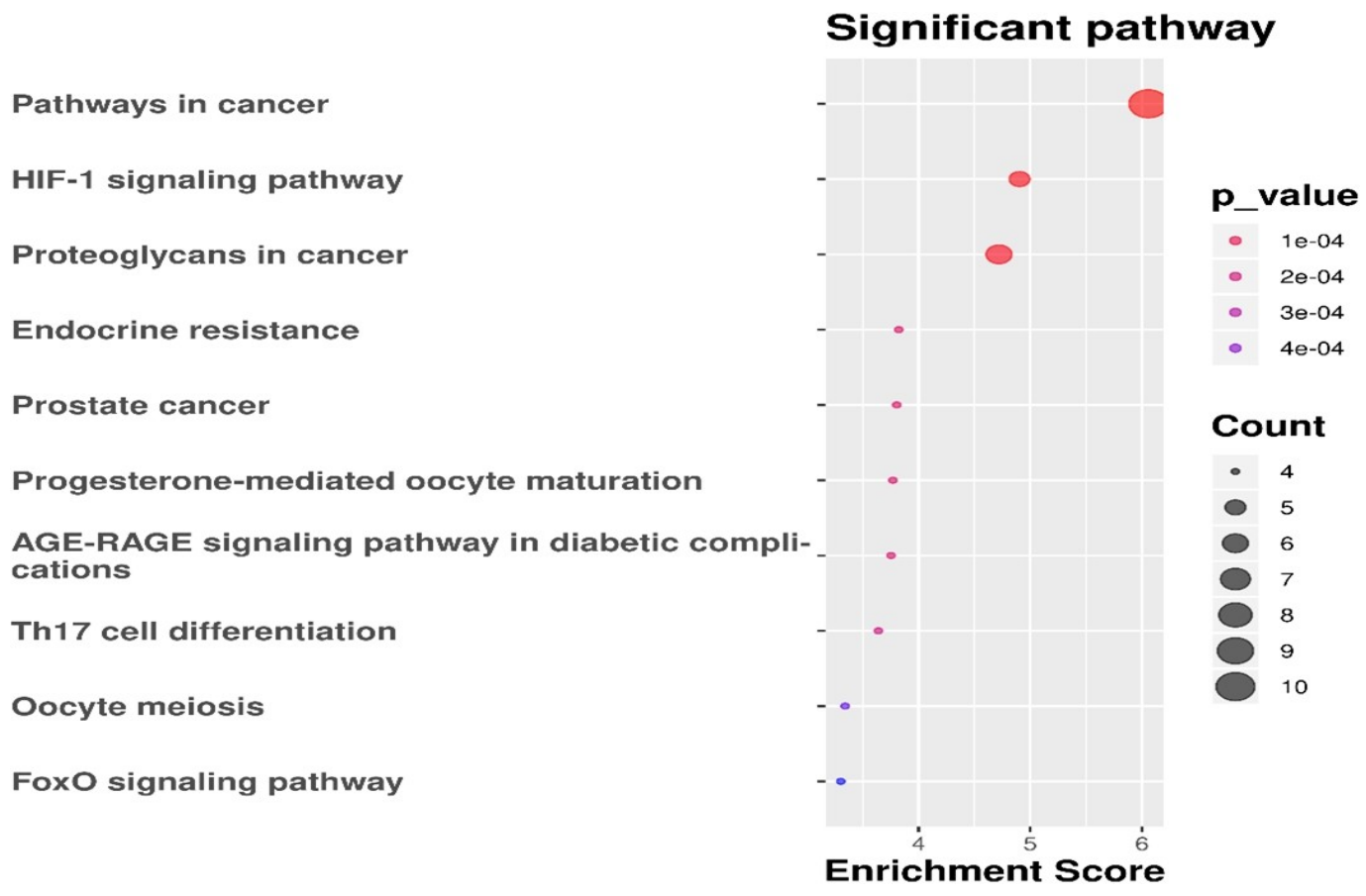

Figure 10 Bubble chart of KEGG enrichment analysis

The pathview package was used to visualize some of the significantly enriched pathways, and the following pictures were obtained (Figure 11): 


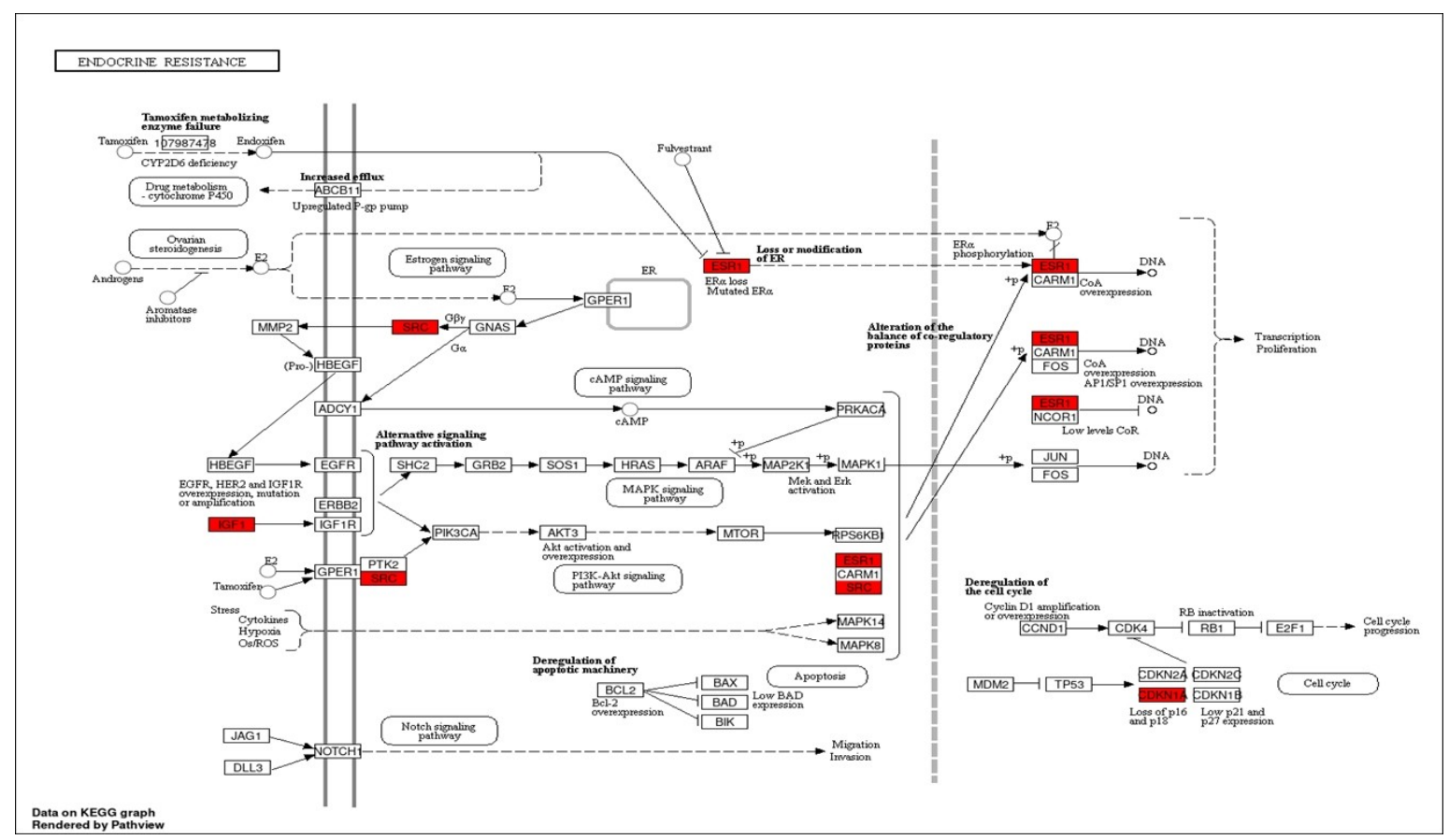

Figure 11 Endocrine resistance signaling pathway

\section{Survival Analysis}

Survival analysis of 23 key target genes was performed using the clinical data and mRNA expression data obtained from the TCGA-CESC project, respectively. The results showed that the expression of $0 / 4$ genes have significantly affected the diseasefree survival rate/overall survival rate. An example of gene survival difference analysis was as follows (Figure 12, Figure 13): 


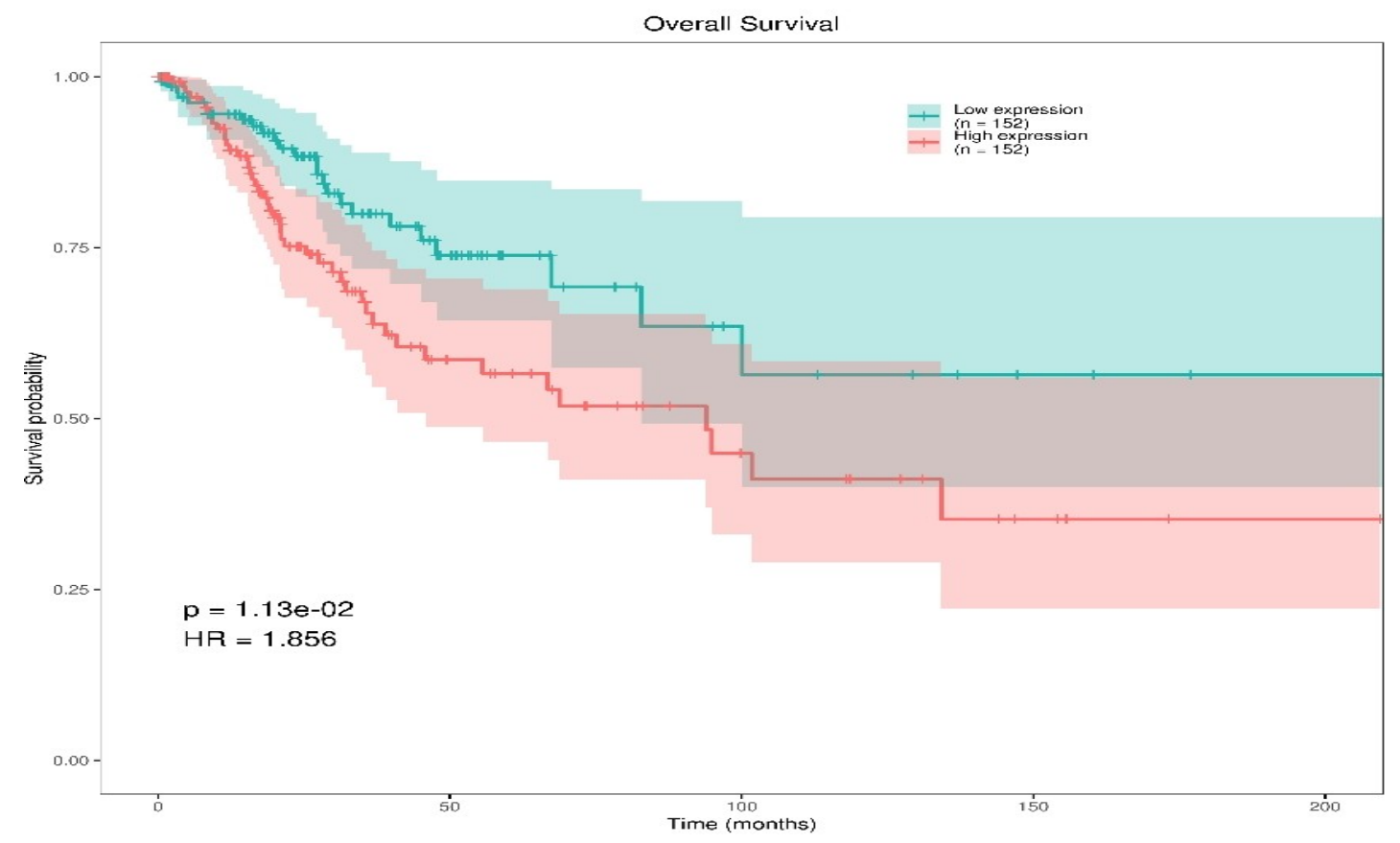

Figure 12 Difference analysis of overall survival rate of SERPINE1

\begin{tabular}{lrrr} 
Variable & $\mathrm{HR}$ & $95 \% \mathrm{Cl}$ & P_value \\
\hline SERPINE1 & 1 & $0.999-1$ & 0.287 \\
years_to_birth & 1.01 & $0.96-1.05$ & 0.816 \\
pathology_T_stage & 1.41 & $0.621-3.18$ & 0.413 \\
pathology_N_stage & 1.58 & $0.365-6.88$ & 0.539 \\
pathology_M_stage & $2.31 \mathrm{e}-08$ & $0-\mathrm{Inf}$ & 0.998 \\
race & 0.774 & $0.493-1.22$ & 0.268 \\
Tumor_purity & 9.48 & $0.0864-1040$ & 0.348 \\
number_of_lymph_nodes & 1.09 & $0.93-1.28$ & 0.286 \\
& & & 1000
\end{tabular}

Figure 13 Multivariate regression forest plot of SERPINE1

\section{Experimental}

1. The purpose of the experiment

Hela cells were added with different concentrations of drug-containing serum for 24 hours to detect the invasion ability of the cells.

2. Experimental materials 
(1) Cervical cancer cell Hela: Cell Resource Center, Institute of Basic Medicine, Chinese Academy of Medical Sciences;

(2) DMEM medium: Gibco company, article number: C11995500BT;

(3) Fetal bovine serum FBS, article number: 04-001-1ACS (BI company);

(4) Penicillin Sodium: Item No.: 69-57-8 (Dalian Meilun Biology);

(5) Streptomycin sulfate: Item No.: 3810-74-0 (Dalian Meilun Biology);

(6) The remaining reagents are all domestically produced analytical grades.

(7) Carbon dioxide incubator: Model: Model 3111 (U.S. Thermo Fisher

Scientific Company);

(8) Inverted phase contrast microscope: ECLIPSE Ti-S type (Japan Nikon Company);

(9) Low-speed automatic balance centrifuge: TDZ4-WS type, Hunan Xiangyi;

(10) Cell culture dishes/cell culture plates: Fisher Scientific, USA;

3. Experimental steps

\subsection{Cell culture}

Cervical cancer cell Hela was cultured in DMEM medium containing 10\% BI in a $37{ }^{\circ} \mathrm{C}, 5 \% \mathrm{CO} 2$ saturated humidity incubator, washed with phosphate buffered saline (PBS), $0.25 \%$ Trypsin $+0.02 \%$ EDTA digested cells Perform subculture and adjust cell status.

\subsection{Cell Plating and Dosing}

Take the normal cultured Hela cell line in the log phase, trypsinize and collect it by centrifugation, make a cell suspension and count the cells, inoculate it into a 
$100 \mathrm{~mm}$ culture dish at $1 \times 10$ cells/well, and wait until the cells grow to $80 \%-85 \%$. , Add Cervical Recipe (mass concentration are 20, 40, 80umol $・$ L-1), the control group is added DMEM medium, and 2 multiple holes are set at the same time. After culturing for 24 hours, collect the cells of each group and extract the total protein of the cells. Perform SDS. PAGE electrophoresis, transfer membrane, blocking, incubate the primary antibody at $4^{\circ} \mathrm{C}$ overnight. After washing with TBST, add the secondary antibody and incubate at room temperature (dilution ratio 1:5000) for 1 hour, wash the membrane with TBST, and finally chemiluminescence, gel imaging and analysis.

\subsection{Cell invasion}

Cell invasion: Dissolve Matrigel in a refrigerator at $4{ }^{\circ} \mathrm{C}$ overnight, dilute Matrigel with serum-free cell culture medium to $300 \mu \mathrm{l} / \mathrm{ml}$ at $4{ }^{\circ} \mathrm{C}$, and apply $100 \mu 1$ evenly on the PET film surface of the cell culture tank, Then gently put the chamber into the wells of the 24-well plate, place it at $37^{\circ} \mathrm{C}$ for about 3 hours, and take it out to dry on an ultra-clean bench overnight.

After the drug treatment, the cells were digested and centrifuged with $0.25 \%$ Trypsin $+0.02 \%$ EDTA, resuspended in serum-free medium, counted, and invaded into groups of $2 \times 104 /$ well/density and spread $150 \mathrm{ul}-250 \mathrm{ul}$ in the chamber on a $24-$ well plate. Add 500ul-600ul culture medium containing $10 \%$ serum to the chamber and place it in a $37^{\circ} \mathrm{C} 3$ incubator overnight.

After overnight incubation, wash 3 times with $1 \times$ PBS, fix with $4 \%$ paraformaldehyde for $15 \mathrm{~min}$ at room temperature, then wash 3 times with $1 \times \mathrm{PBS}$, wipe the upper chamber cells with cotton swab, add crystal violet for staining for 
$15 \mathrm{~min}$, wash with $1 \times$ PBS 3 Then, place it in air-dry at room temperature and take pictures under a microscope.

The experiment was repeated three times.

4. Experimental results

\subsubsection{Inhibition of HeLa cell invasion}

After 24 hours of different concentrations of the medicine-containing serum of the Cervical Recipe acted on HeLa cells, compared with the blank group, the cell invasion ability of the model group was significantly reduced $(\mathrm{P}<0.01)$, indicating that Cervical Recipe has a significant inhibitory effect on the invasion ability of HeLa cells And the higher the concentration, the more obvious the effect of inhibiting cell invasion (Table 5, Figure 14).

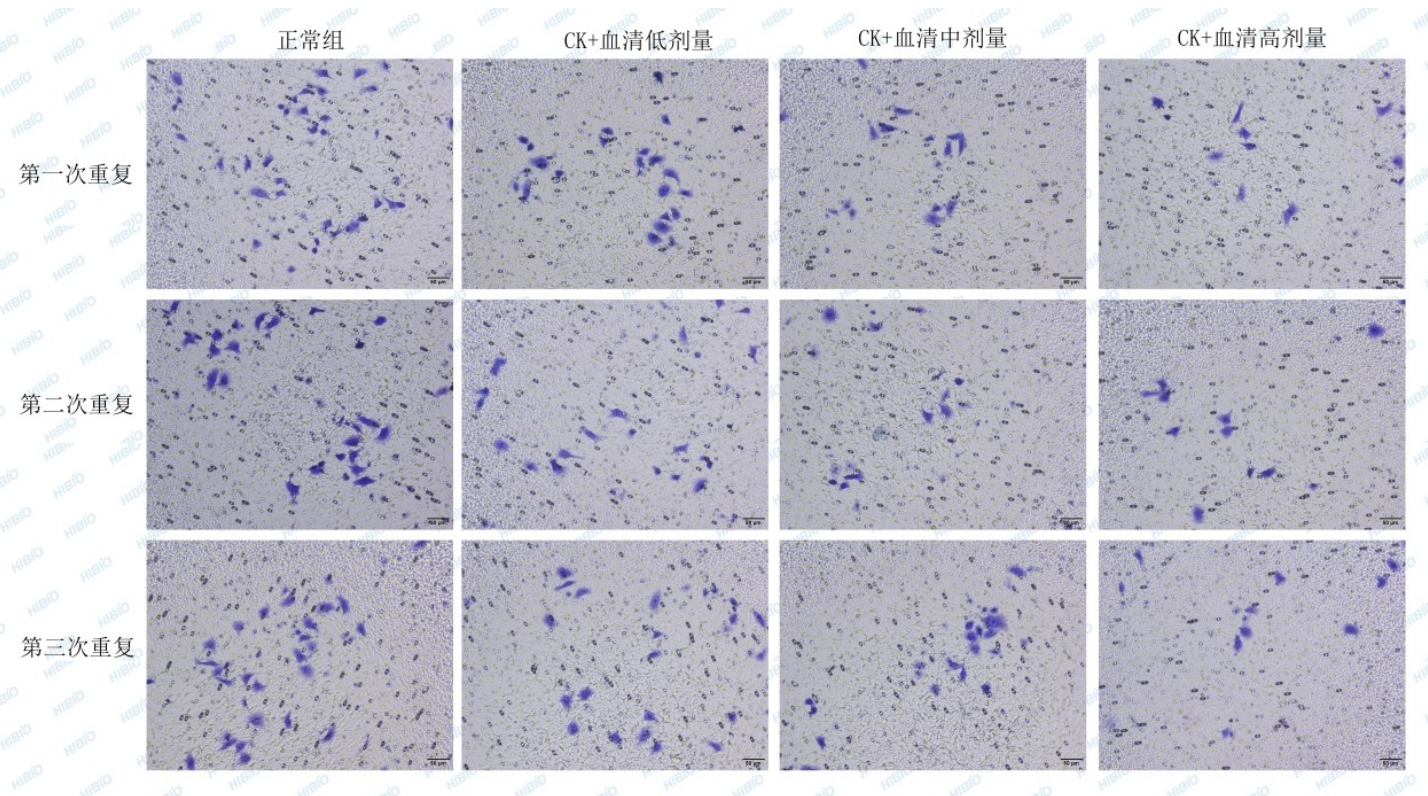

Figure 14 Cell statistics

\section{Discussion}

There is no record on cervical cancer and HPV infection in TCM, but the category of

"carrying disease" and "sickness" can be attributed according to its clinical 
manifestations such as large amount and abnormal color and taste. "Carry down disease" involves a summary of abnormal diseases caused by vaginitis, cervicitis, pelvic inflammatory disease, and genital tumors in western medicine. TCM believes that cancer is an empirical evidence, and it is caused by damp heat and blood stasis toxins assault on the internal content, but cancer also includes internal deficiency. The pathogenesis of cervical cancer involves "lack of righteous qi, the damp heat and evil toxins congregating in the Zimen". The most common type of cervical cancer syndrome differentiation involves damp toxins. The disease is located in any region of the cervix and the second vein. The treatment requires "clearing heat, detoxification, and attacking". The most method used involves "dispersion of poison and strengthening the body". The founder of Cervical Prescription, the Chief Physician Qiu Xiaomei, is in the first batch of national-level veteran traditional Chinese medicine practitioners and is the recipient of State Council allowances. He has been engaged in clinical teaching, treatment and scientific research of Chinese medicine for more than 50 years.

According to many years of clinical experience, Doctor Qiu believed that the main cause of the disease involves dampness. Although dampness is a pathogenic condition, it causes invasion and transformation of dampness, but it lies in the imbalance of the liver, spleen and kidney functions in the human body. All these pathologies and exogenous damp toxins might infiltrate and carry into the veins along the meridians. In the occurrence of cervical cancer, inflammation and damage of the cervical epithelium are the early stages of HPV infection and precancerous lesions. 
The displacement of cervical squamous-column junction changes with the menstrual cycle and hormone levels, and the erosion-like changes in the transformation zone are almost considered inevitable. Therefore, how to ensure the integrity of the cervical epithelium is an important measure to prevent HPV infection and cervical cancer. For patients with cervicitis and HPV infection, and before they develop into cervical cancer, TCM with the philosophy of "preventing the disease before the disease should be applied to prevent the change of the existing disease". Based on the syndrome differentiation and treatment, the combination of internal and external treatment can effectively control the condition. To improve the prognosis, the precancerous lesions of the cervix are mainly treated locally due to special location.

The external treatment of TCM not only involves surface absorption of effective ingredients of the medicine, but they also can dredge the meridians and regulate the qi by stimulating the medicine to the specific part. This can be easily operated and is a relatively safe method for treating cervical cancer. No damage occurs to the gastrointestinal tract, and there is little impact on liver and kidney function. Catechu, borneol, alum, and other medicines when used in combination can promote local blood circulation, and improve tissue metabolism in the diseased area, thereby weakening the local pathological reactions, eliminating discomfort, anti-inflammatory, analgesic, and reducing edema. In contrast, modern medicine uses laser, freezing, electric ironing, leap surgery, etc., which have certain advantages. After physical therapy and surgical treatment, vaginal discharge, watery discharge, irregular vaginal bleeding, and cervical scars might often occur postoperatively. 
Cervical Prescription is composed of alum, catechu, and borneol. The catechin is cool in nature, bitter in taste, and enters the heart and the lung meridians. According to the "Compendium of Materia Medica", catechu is used for "coating golden sores, all kinds of sores, generating muscles and alleviating pain, stopping bleeding, and hygroscopic." Modern pharmacological research showed that catechu contains catechin tannin, catechin, quercetin and other ingredients. The main ingredient is the catechin tannin, which accounted for $20 \%-50 \%$ of the catechu. It has inhibitory effects on Staphylococcus aureus, Candida albicans, Pseudomonas aeruginosa and other bacteria, and has certain analgesic and anti-tumor effects (16).

Borneol is slightly cold in nature, pungent in taste, and targets Guixin, spleen and lung channels. "The Synopsis of the Golden Chamber" involves the use of borneol treatment for itching. The main ingredients of borneol include borneol and isoborneol. Tests have proved that borneol has obvious antibacterial effects on Staphylococcus aureus, Streptococcus, Escherichia coli, etc. (17).

Alum is sour, cold in nature, and used as astringent. It reaches to the lung, liver, spleen, and large intestine meridian. According to "The Classic": "Alum is mainly for cold and heat to release diarrhea, white womb, erosive, eye pain, hard bones and teeth." Alum mainly contains potassium sulphates. The tests showed that this product has an obvious inhibitory effects on a variety of Gram-positive bacteria. Alum liquid acts as a powerful astringent and causes damp-drying. When it meets the protein, it becomes a poorly soluble compound and then precipitates (18). In this recipe, it mainly adheres to a moisture absorbent. 
The key target network of Cervical Decoction was found in the treatment of cervical cancer, in which the HIF-1 signaling pathway, endocrine resistance, proteoglycan in cancer, and Th17 cell differentiation, are considered as important gene targets, and these genes are all related to the regulation of immune system, which directly participates in tumor regulation pathway. GO analysis results showed that the biological processes involved in the enrichment of key gene targets include secretory granule lumen, cytoplasmic vesicle lumen, vesicle lumen, secretory granules, secretory vesicles, platelet alpha granule lumen, lysosomes, and platelets Alpha granule, cytoplasmic perinuclear cell, enzyme binding, sign receptor binding, heme binding, protein kinase binding, regulation of cell death, negative regulation of cell death, regulation of apoptosis process, regulation of programmed cell death, procedural Negative regulation of cell death.

It shows response to lipids, enzyme binding, organ-activated transcription factor activity, steroid hormone receptor activity, nitric oxide synthase modulator activity, etc. Enrichment analysis of KEGG pathway showed that the signaling pathways associated with Qiu's Cervical Prescription in the treatment of cervical cancer include proteoglycan, HIF-1 signaling pathway, hsa01522pathview, hsa04114pathview, hsa04659pathview, hsa04914pathview, hsa04933pathview, hsa05200pathview, hsa05205pathview, hsa05215 pathway, etc. Studies have confirmed that hypoxiainducible factor-1 (HIF-1), which is a highly specific nuclear transcription factor, plays an important role in the process of cell perception and adaptation to changes in internal environmental oxygen pressure (19). Several studies have shown 
that HIF-1 $\alpha$ has physiological effects such as promoting angiogenesis, regulating internal environmental $\mathrm{pH}$, inducing autophagy and programmed cell death, and promoting self-renewability and differentiation of mesenchymal stem cells, and it is often found in a variety of primary. The expression level of HIF-1 $\alpha$ in secondary malignant tumor tissues is shown to be abnormally increased (20). The proangiogenesis function of HIF-1 is also involved in the remodeling of tumor microenvironment, indirectly promoting proliferation, metastasis of tumor cells and sensitivity to radiotherapy as well as chemotherapy (21).

In summary, this study found that Qiu's Cervical Prescription mainly uses PTGS2, IGF1, ESR1, SRC, HIF1A, IL1B and other gene targets through network pharmacology, and regulates cancer pathways, FoxO signaling pathway, IL-17 and other signaling pathways to regulate immunity and improve cervical cell pathology in patients with cervical cancer, thereby exerting therapeutic effects of cervical cancer.

\section{Conclusion}

This study uses network pharmacology to explore the intervention of Qiu's Cervical Prescription on cervical cancer, which is reflected with the formation of cervical cancer, tumor cell metabolism, immune regulation, cell proliferation, apoptosis, and tumor resistance. During the early stage of cervical cancer, the formation of cervical cancer microenvironment can be adjusted to delay the carcinogenic process; and during the chemotherapy resistance period, the body hormones and tumor cell metabolism can be adjusted to increase tumor cell sensitivity and reverse the transformational therapy resistance. This also shows that the intervention of TCM for 
clearing heat and dampness and detoxification on cervical cancer is multi-target, multi-channel, and involves a systemic regulatory process. However, due to limitations in the conditions, this study also has many shortcomings, such as failed to integrate the concept of "junchen adjuvant" in TCM prescriptions, the impact of dose relationship in compound interventions in diseases, the lateness of data update, scientific research, the accuracy of network pharmacology, etc., leading to uncertainty of the prediction results of network pharmacology to a certain extent. So, the results should still be verified in a relevant basic research.

\section{References}

[1] Marth C, Landoni F, Mahner S,McCormack M, Gonzalez-Martin A, Colombo N. Committee EG. Cervical cancer: ESMO Clinical Practice Guidelines for diagnosis, treatment and follow-up [published correction appears in Ann Oncol. 2018 Oct 1;29(Suppl 4):iv262] [published correction appears in Ann Oncol. 2018 Oct;29 Suppl 4:iv262]. Ann Oncol. 2017;28(suppl_4):iv72-iv83.doi:10.1093/annonc/mdx220.

[2]DeSantis CE, Miller KD, Goding Sauer A, Jemal A, Siegel RL. Cancer statistics for African Americans, 2019. CA Cancer J Clin 2019.DeSantis CE, Miller KD, Goding Sauer A, Jemal A, Siegel RL. Cancer statistics for African Americans, 2019. CA Cancer J Clin.2019;69(3):211-233. doi:10.3322/caac.21555.

[3]Shi Yifu.Related issues of cervical human papillomavirus infection[J].China Family Planning and Obstetrics and Gynecology7(12):1-3,2015,(In Chinese). [4]Di Paola M, Sani C, Clemente AM, et al. Characterization of cervico-vaginal microbiota in women developing persistent high-risk Human Papillomavirus infection. Sci Rep. 2017;7(1):10200. Published 2017 Aug 31. 
doi:10.1038/s41598-017-09842-6.

[5] Hopkins AL. Network pharmacology: the next paradigm in drug discovery. Nat Chem Biol. 2008;4(11):682-690. doi:10.1038/nchembio.118.

[6] Liu Z, Guo F, Wang Y, et al. BATMAN-TCM: a Bioinformatics Analysis Tool for Molecular mechANism of Traditional Chinese Medicine. Sci Rep. 2016;6:21146. Published 2016 Feb 16. doi:10.1038/srep21146.

[7] Ogata H, Goto S, Sato K, Fujibuchi W, Bono H, Kanehisa M. KEGG: Kyoto Encyclopedia of Genes and Genomes. Nucleic Acids Res. 1999;27(1):29-34. doi:10.1093/nar/27.1.29

[8] Thorn CF, Klein TE, Altman RB. PharmGKB: the Pharmacogenomics Knowledge Base. Methods Mol Biol. 2013;1015:311-320. doi:10.1007/978-1-62703-435-7_20 [9] Davis AP, Grondin CJ, Johnson RJ, et al. The Comparative Toxicogenomics Database: update 2019. Nucleic Acids Res. 2019;47(D1):D948-D954. doi:10.1093/nar/gky868.

[10] Safran M, Dalah I, Alexander J, et al. GeneCards Version 3: the human gene integrator. Database (Oxford). 2010;2010:baq020. Published 2010 Aug 5. doi:10.1093/database/baq020.

[11] Piñero J, Ramírez-Anguita JM, Saüch-Pitarch J, et al. The DisGeNET knowledge platform for disease genomics: 2019 update. Nucleic Acids Res. 2020;48(D1):D845-D855. doi:10.1093/nar/gkz1021 
[12] Shannon P, Markiel A, Ozier O, et al. Cytoscape: a software environment for integrated models of biomolecular interaction networks. Genome Res. 2003;13(11):2498-2504. doi:10.1101/gr.1239303.

[13] Von Mering C, Huynen M, Jaeggi D, Schmidt S, Bork P, Snel B. STRING: a database of predicted functional associations between proteins. Nucleic Acids Res. 2003;31(1):258-261. doi:10.1093/nar/gkg034 .

[14] Chin CH, Chen SH, Wu HH, Ho CW, Ko MT, Lin CY. cytoHubba: identifying hub objects and sub-networks from complex interactome. BMC Syst Biol. 2014;8 Suppl 4(Suppl 4):S11. doi:10.1186/1752-0509-8-S4-S11.

[15] Ashburner M, Ball CA, Blake JA, et al. Gene ontology: tool for the unification of biology. The Gene Ontology Consortium. Nat Genet. 2000;25(1):25-29. doi:10.1038/75556.

[16] Meng Fanjia,Liu Lei,Zhang Wenwei,Wu Lihong,Fu Qifeng,Kang Yuhong,Zhang Yanli.The research progress of catechu[J].Science and Technology Innovation03:45-46,2020(In Chinese).

[17] Li Qianjun,Hu Yilin,Liang Yan,Wang Ting,Wu Xiaobin, Chao Yan.Effects of Baofukang Suppository on the proliferation and apoptosis of cervical cancer cell $\mathrm{SiHa}$ in vitro[J].International Journal of Laboratory Medicine40(01):16-18,2019(In Chinese).

[18] Hao Jian,Wu Xiongzhi.The effect of alum on the proliferation of HepG2 and SMMC-7721 liver cancer cells and its mechanism[J].Shandong Medicine55(23),2015(In Chinese). 
[19] Wang XJ, Si LB. Advances on hypoxia inducible factor-1. Chin Med J (Engl). 2013;126(18):3567-3571.

[20] Tuomisto A, García-Solano J, Sirniö P, et al. HIF-1 $\alpha$ expression and high microvessel density are characteristic features in serrated colorectal cancer. Virchows Arch. 2016;469(4):395-404. doi:10.1007/s00428-016-1988-8.

[21] Du Y, Long Q, Zhang L, Shi Y, Liu X, Li X, Guan B, Tian Y, Wang X, Li L, He D. Curcumin inhibits cancer-associated fibroblast-driven prostate cancer invasion through MAOA/mTOR/HIF-1 $\alpha$ signaling. Int J Oncol. 2015 Dec;47(6):2064-72. doi: 10.3892/ijo.2015.3202. Epub 2015 Oct 13. PMID: 26499200; PMCID: PMC4665143. 
Figures
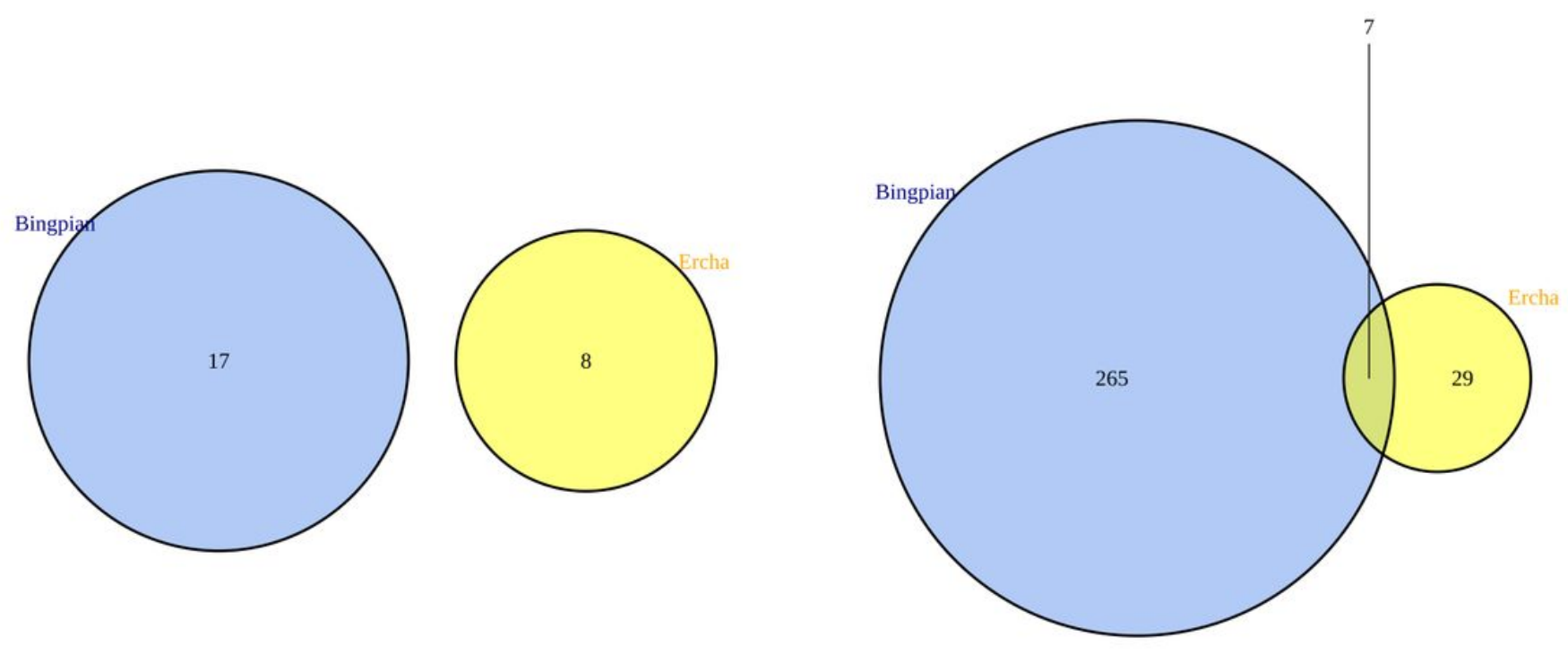

Figure 1

Interaction target intersection and union Venn diagram 


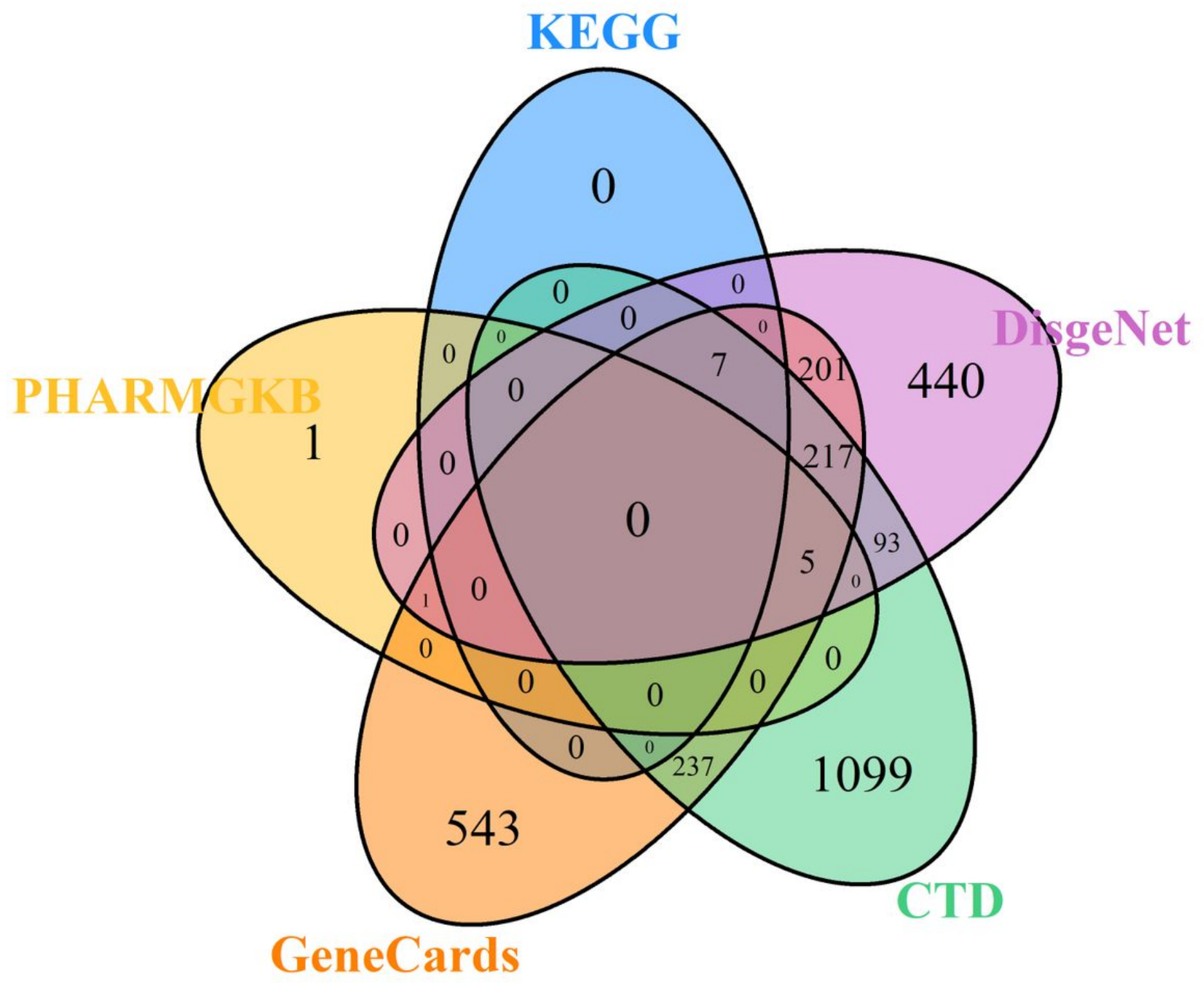

Figure 2

Five Potential cervical cancer targets in databases 


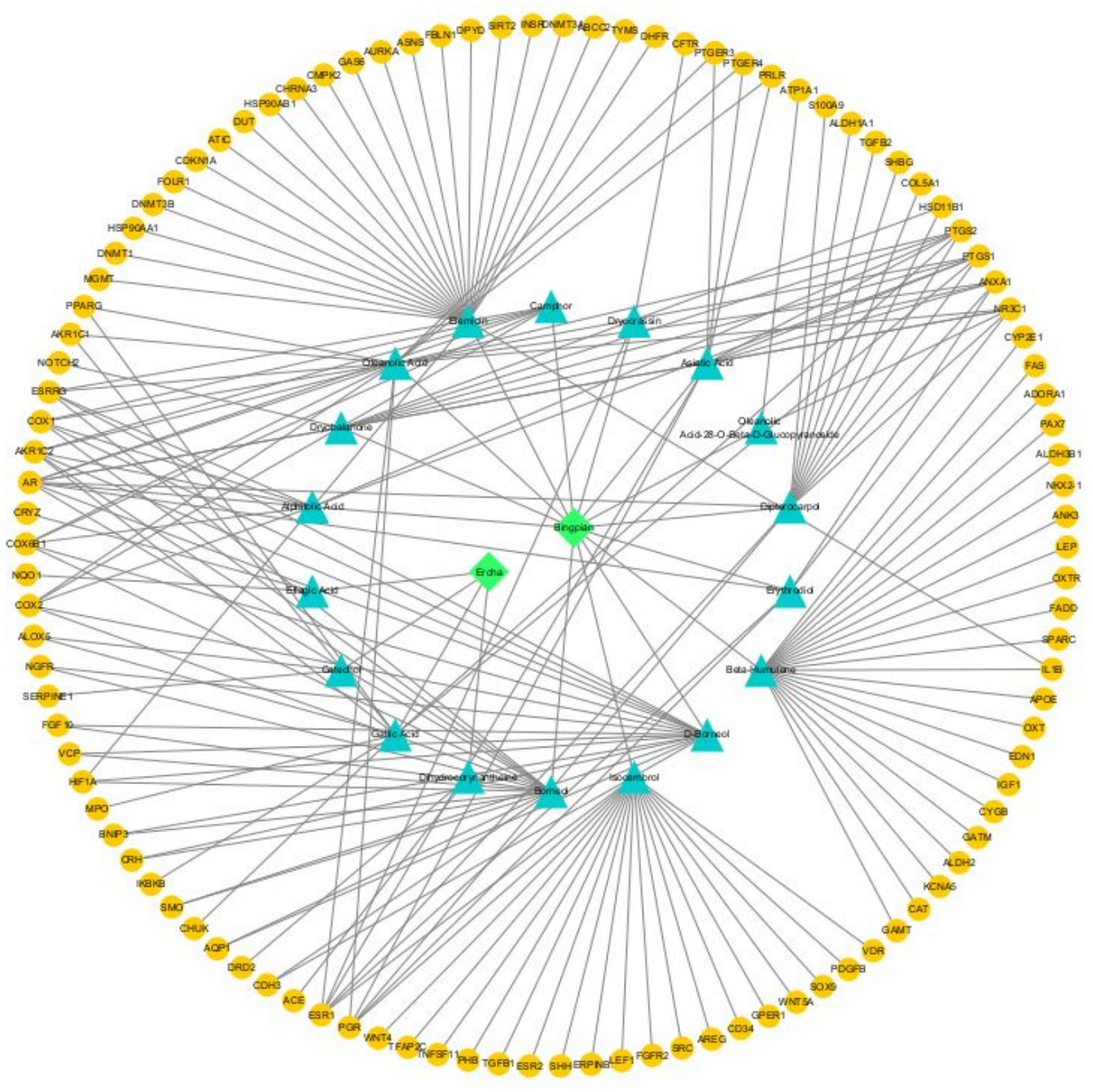

Figure 3

Traditional Chinese Medicine Compound-Ingredients-Target Network 


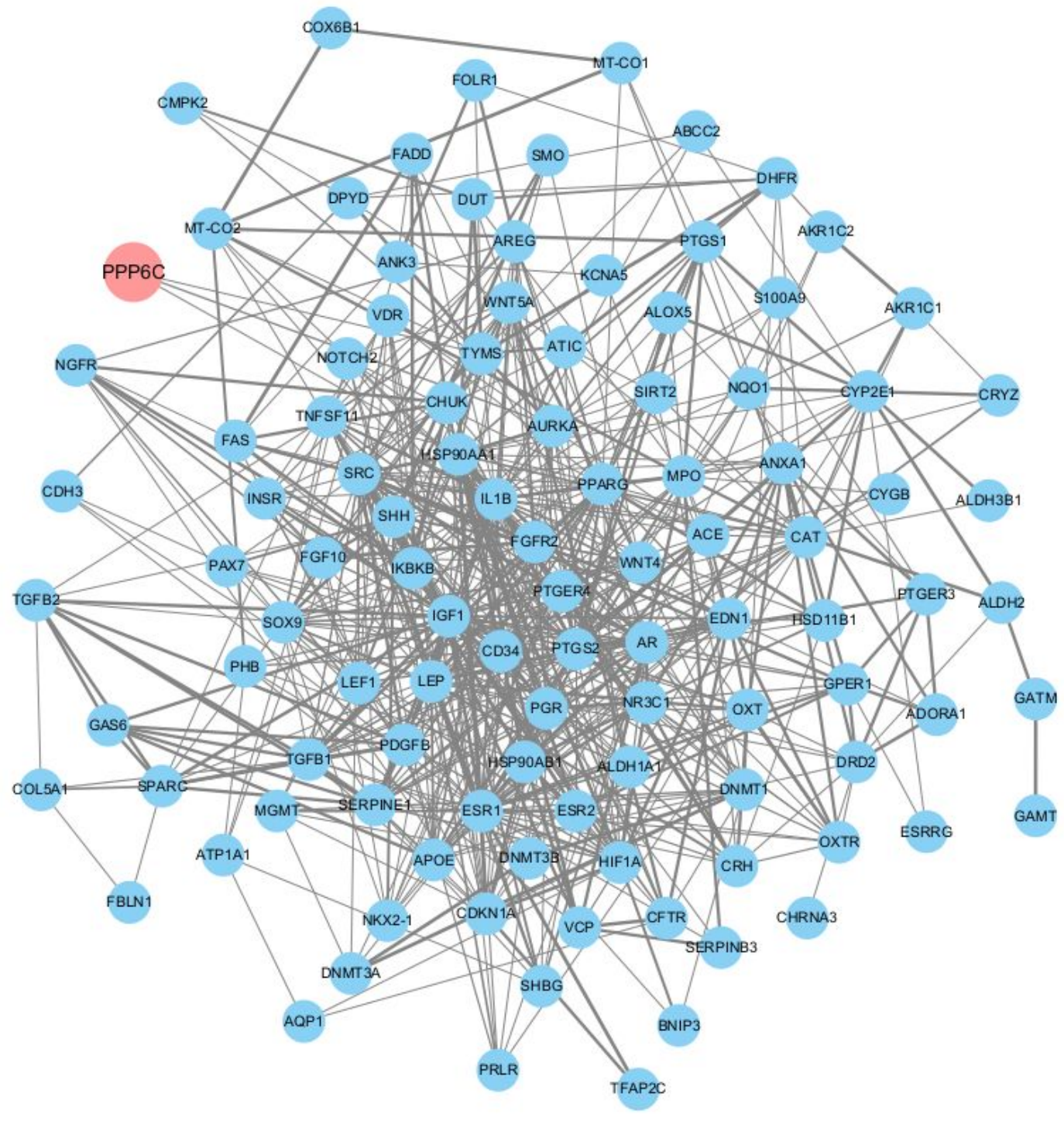

Figure 4

PPI interaction network of cervical cancer target protein 


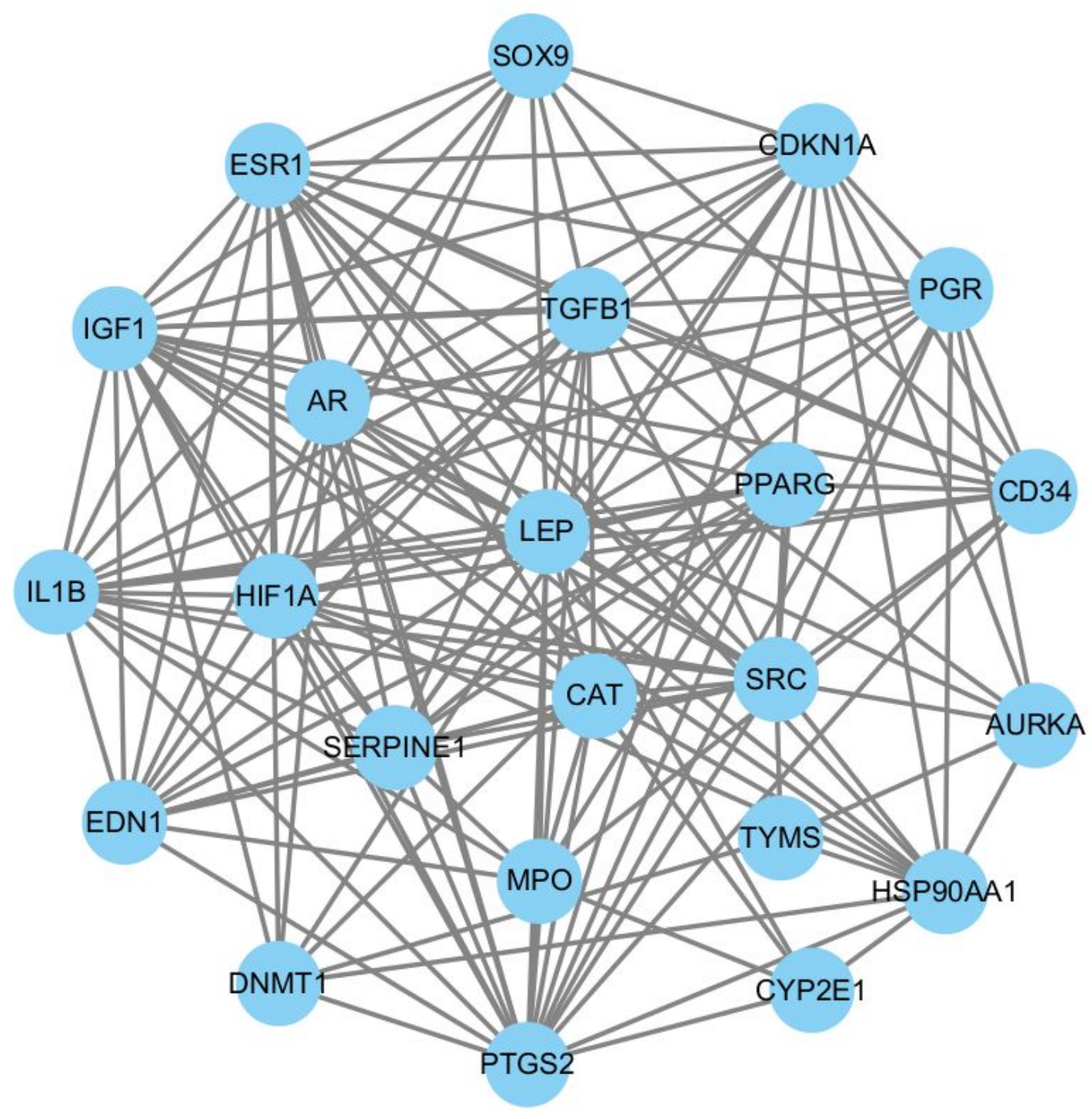

Figure 5

PPI interaction network of key target proteins of the compound 


\section{Significant GO-BP}

Response to organic substance

Response to lipid

Response to organic cyclic compound

Cellular response to organic substance

Response to hormone

Regulation of cell death

Negative regulation of cell death

Response to drug

Response to steroid hormone

Positive regulation of metabolic process

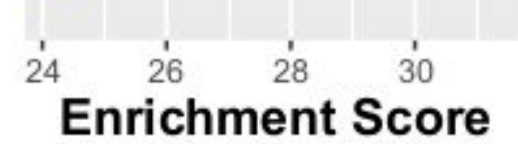

\section{p_value}

- $3.837407 e-32$

- $2.044314 \mathrm{e}-25$

- $4.088628 \mathrm{e}-25$

- $6.132943 \mathrm{e}-25$

- $8.177257 e-25$

\section{Count}

- 30

- 40

50

60

70

\section{Figure 6}

GO enrichment analysis of genes in the network of "Chinese medicine compound-components-targets": bubble chart. 


\section{Significant pathway}

Pathways in cancer

Antifolate resistance

Prostate cancer

Proteoglycans in cancer

Breast cancer

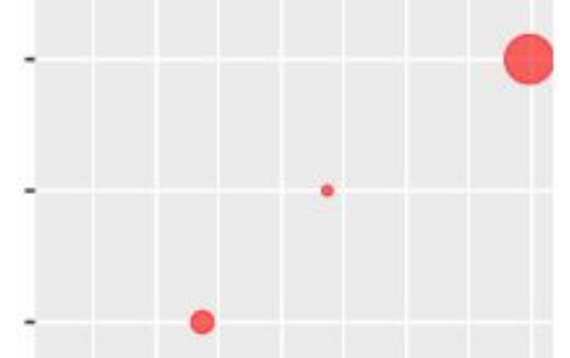

p_value

- $1 \mathrm{e}-05$

- $2 \mathrm{e}-05$

- $3 e-05$

Chagas disease (American trypanosomiasis) -

Count

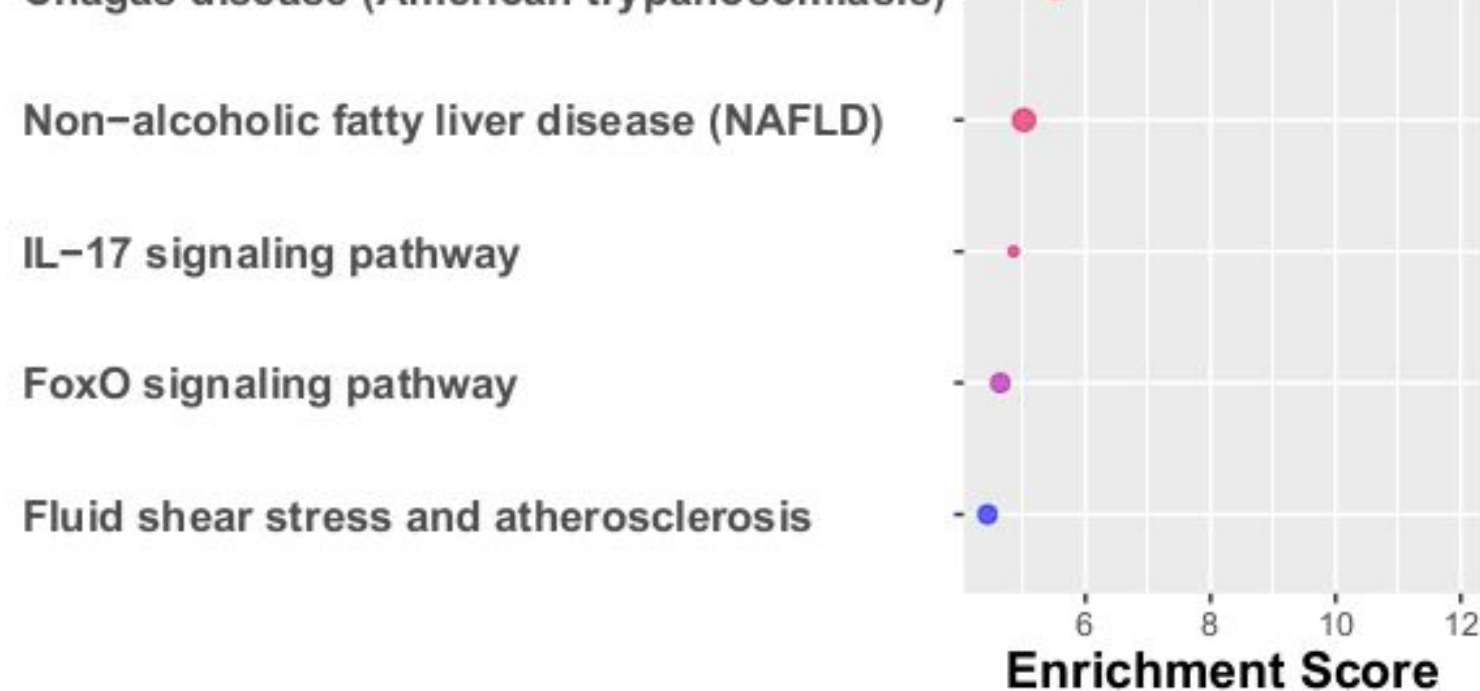

- 10

- 15

- 20

25

Enrichment Score

\section{Figure 7}

KEGG enrichment analysis of genes in the network of "Chinese medicine compound-components-targets": bubble chart 


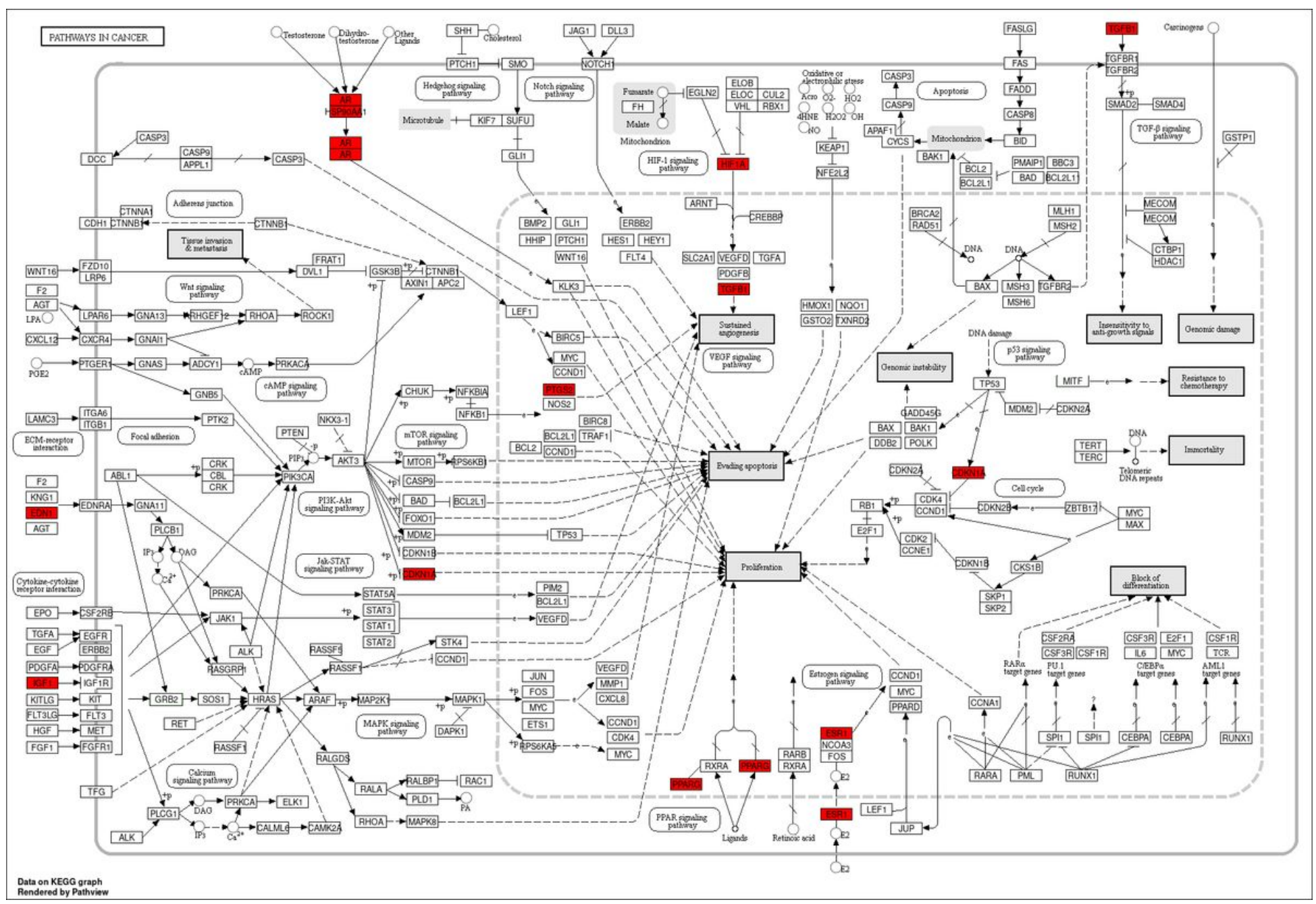

Figure 8

Pathway in cancer 


\section{Significant GO-BP}

Response to lipid

Regulation of cell death

Negative regulation of cell death

Regulation of apoptotic process

Regulation of programmed cell death

Tube development

Negative regulation of apoptotic process

Negative regulation of programmed cell d- . eath

Response to organic substance

Regulation of reactive oxygen species me- . . tabolic process

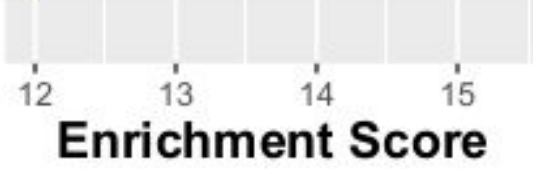

- 9

- 12

15

- $2.5 \mathrm{e}-13$

- $5.0 \mathrm{e}-13$

- $7.5 \mathrm{e}-13$

- $1.0 \mathrm{e}-12$

\section{Count}

2
15
18
21
1 
Pathways in cancer

Antifolate resistance

Prostate cancer

Proteoglycans in cancer

Breast cancer

Chagas disease (American trypanosomiasis) -

Non-alcoholic fatty liver disease (NAFLD)

IL-17 signaling pathway

FoxO signaling pathway

Fluid shear stress and atheroscleros is

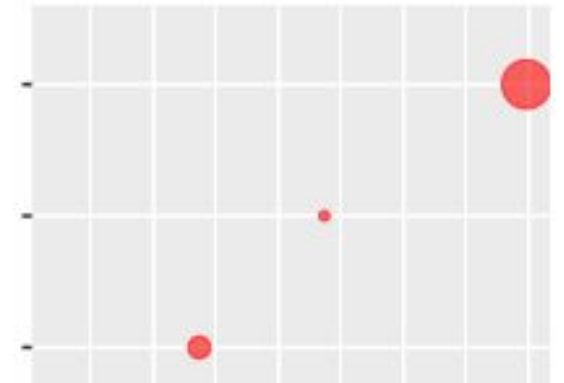

p_value

- $1 \mathrm{e}-05$

- $2 \mathrm{e}-05$

- $3 e-05$

\section{Count}

- 10

- 15

- 20

(1) 25

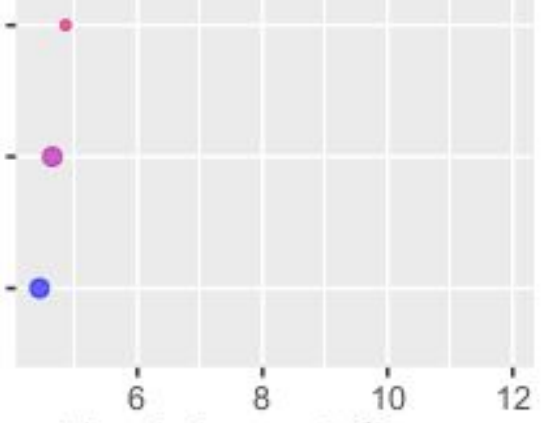

Enrichment Score

Figure 10

Bubble chart of KEGG enrichment analysis 
ENDOCRINE RESISTANCE

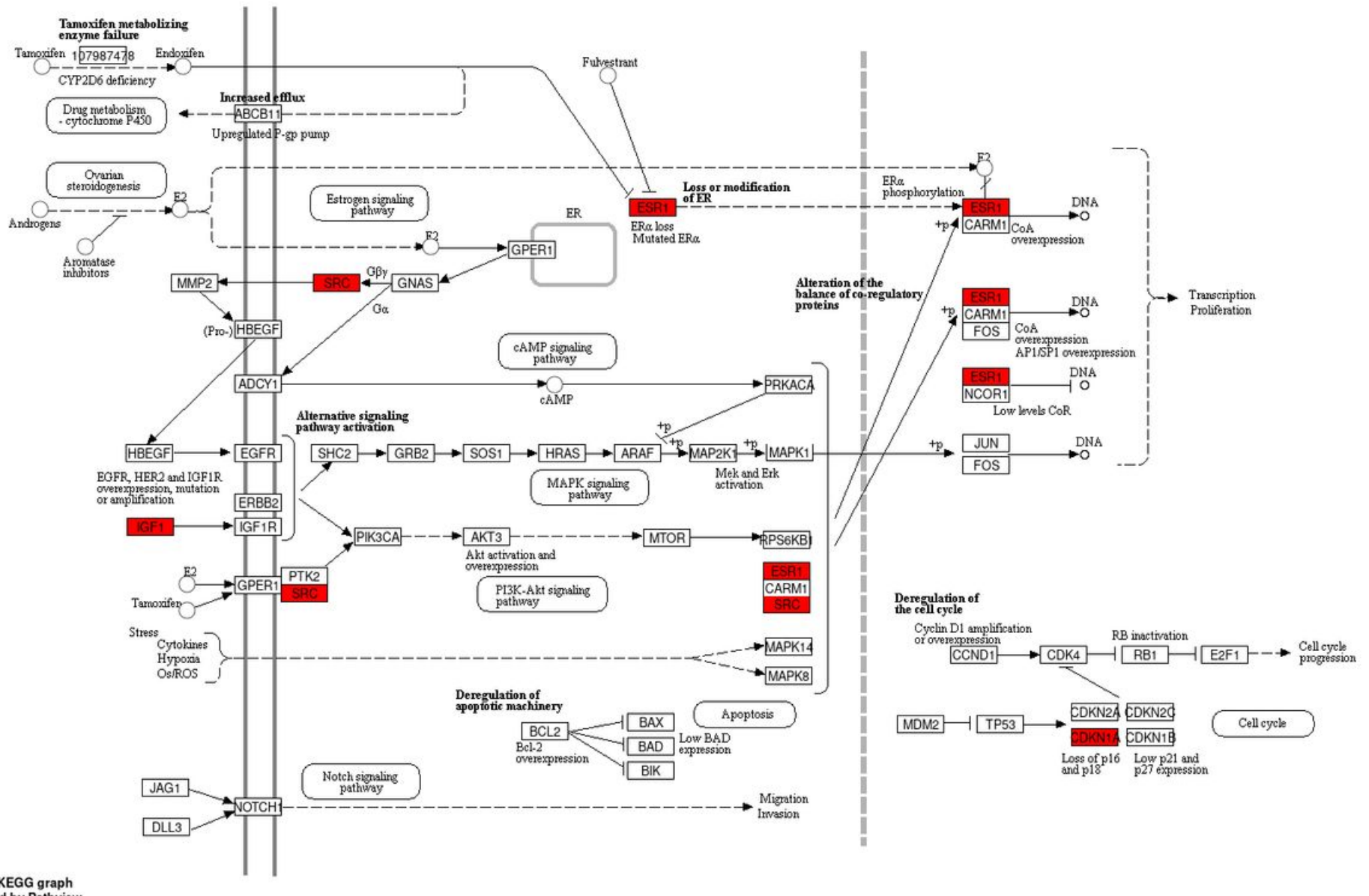

Figure 11

Endocrine resistance signaling pathway 


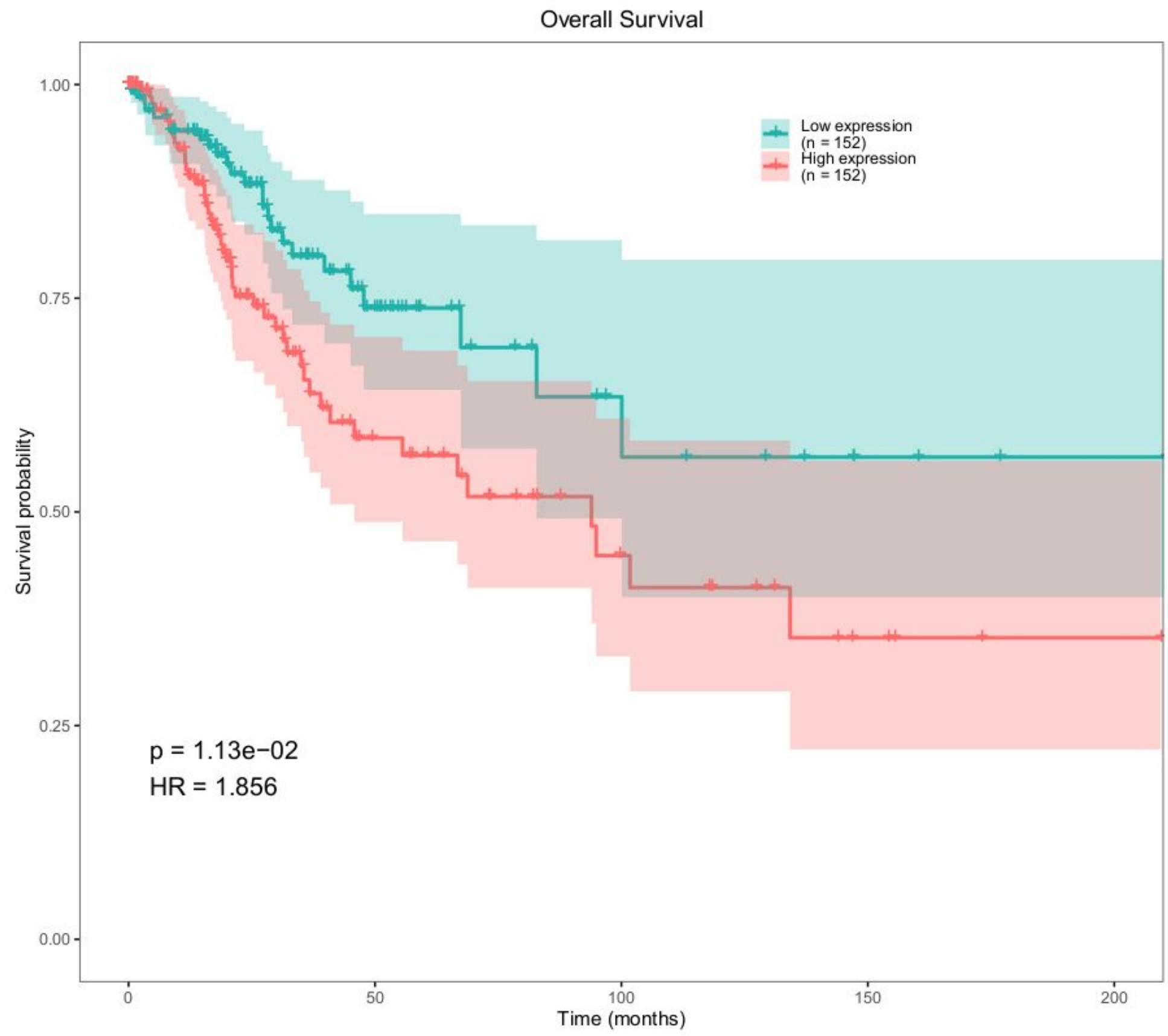

Figure 12

Difference analysis of overall survival rate of SERPINE1 


\begin{tabular}{|c|c|c|c|}
\hline Variable & HR & $95 \% \mathrm{Cl}$ & p_value \\
\hline SERPINE1 & 1 & $0.999-1$ & 0.287 \\
\hline years_to_birth & 1.01 & $0.96-1.05$ & 0.816 \\
\hline pathology_T_stage & 1.41 & $0.621-3.18$ & 0.413 \\
\hline pathology_N_stage & 1.58 & $0.365-6.88$ & 0.539 \\
\hline pathology_M_stage & $2.31 e-08$ & $0-\operatorname{Inf}$ & 0.998 \\
\hline race & 0.774 & $0.493-1.22$ & 0.268 \\
\hline Tumor_purity & 9.48 & $0.0864-1040$ & 0.348 \\
\hline \multirow[t]{2}{*}{ number_of_lymph_nodes } & 1.09 & $0.93-1.28$ & 0.286 \\
\hline & & & \\
\hline
\end{tabular}

Figure 13

Multivariate regression forest plot of SERPINE1

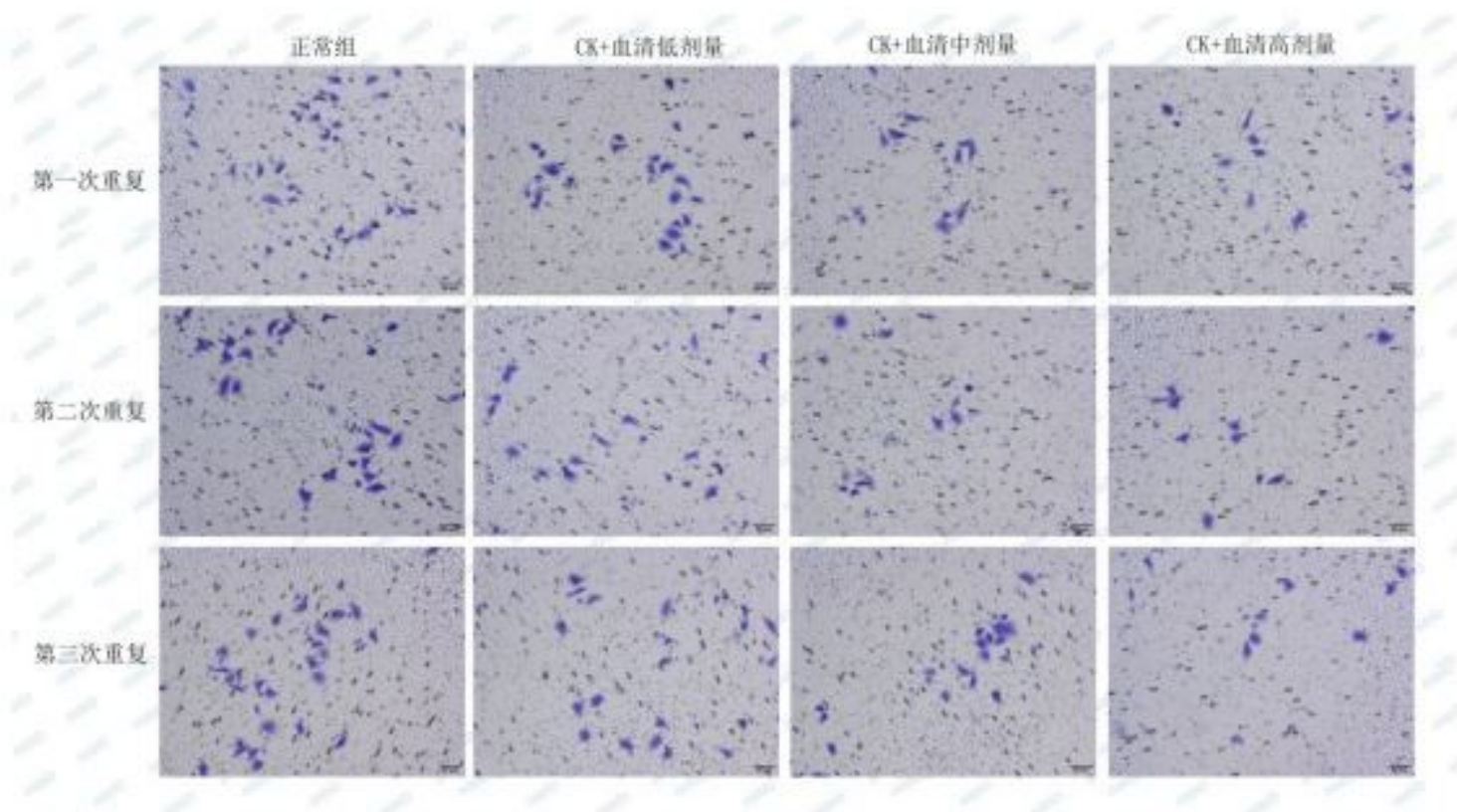

Figure 14

Cell statistics 